\title{
The complex planetary synchronization structure of the solar system
}

\author{
N. Scafetta \\ Active Cavity Radiometer Irradiance Monitor (ACRIM) Lab, Coronado, CA 92118, USA \\ Duke University, Durham, NC 27708, USA \\ Correspondence to: N. Scafetta (nicola.scafetta@gmail.com)
}

Received: 12 December 2013 - Revised: 19 December 2013 - Accepted: 28 December 2013 - Published: 15 January 2014

Abstract. The complex planetary synchronization structure of the solar system, which since Pythagoras of Samos (ca. 570-495 BC) is known as the music of the spheres, is briefly reviewed from the Renaissance up to contemporary research. Copernicus' heliocentric model from 1543 suggested that the planets of our solar system form a kind of mutually ordered and quasi-synchronized system. From 1596 to 1619 Kepler formulated preliminary mathematical relations of approximate commensurabilities among the planets, which were later reformulated in the Titius-Bode rule (1766-1772), which successfully predicted the orbital position of Ceres and Uranus. Following the discovery of the $\sim 11$ yr sunspot cycle, in 1859 Wolf suggested that the observed solar variability could be approximately synchronized with the orbital movements of Venus, Earth, Jupiter and Saturn. Modern research has further confirmed that (1) the planetary orbital periods can be approximately deduced from a simple system of resonant frequencies; (2) the solar system oscillates with a specific set of gravitational frequencies, and many of them (e.g., within the range between $3 \mathrm{yr}$ and $100 \mathrm{yr}$ ) can be approximately constructed as harmonics of a base period of $\sim 178.38 \mathrm{yr}$; and (3) solar and climate records are also characterized by planetary harmonics from the monthly to the millennial timescales. This short review concludes with an emphasis on the contribution of the author's research on the empirical evidences and physical modeling of both solar and climate variability based on astronomical harmonics. The general conclusion is that the solar system works as a resonator characterized by a specific harmonic planetary structure that also synchronizes the Sun's activity and the Earth's climate. The special issue Pattern in solar variability, their planetary origin and terrestrial impacts (Mörner et al., 2013) further develops the ideas about the planetary-solar-terrestrial interaction with the personal contribution of 10 authors.

\section{Introduction}

In 1543 the De revolutionibus orbium coelestium (On the Revolutions of the Heavenly Spheres) was published. As opposed to Ptolemy's geocentric model that had been widely accepted since antiquity, Copernicus (1543) proposed a heliocentric model for the solar system: the planets, including the Earth, orbit the Sun and their orbital periods increase with the planetary distance from the Sun. Copernicus also argued that the planets form a kind of mutually ordered system. The physical properties of the planets' orbits, such as their distances from the Sun and their periods, did not appear to be randomly distributed. They appeared to obey a certain law of nature.
A typical synchronization that could be more easily highlighted by the heliocentric system was, for example, the $8: 13$ Earth-Venus orbital resonance. Every 8 yr the EarthVenus orbital configuration approximately repeats because the Earth revolves 8 times and Venus $\sim 13$ times, as can be easily calculated using their sidereal orbital periods: $P_{\mathrm{Ea}}=$ 365.256 days and $P_{\mathrm{Ve}}=224.701$ days. Figure 1a demonstrates this orbital regularity by showing the relative positions of Earth and Venus on 1 January from 2012 to 2020.

However, Venus presents a more subtle and remarkable synchronization with Earth. The rotation period of Venus on its own axis is 243.021 days (that is, almost exactly twothirds of the Earth's annual period) and is retrograde. It is 
A

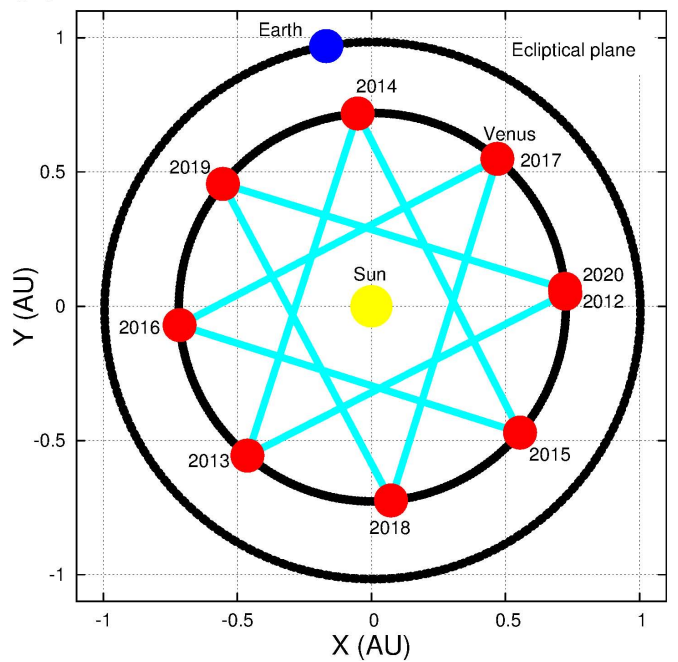

B

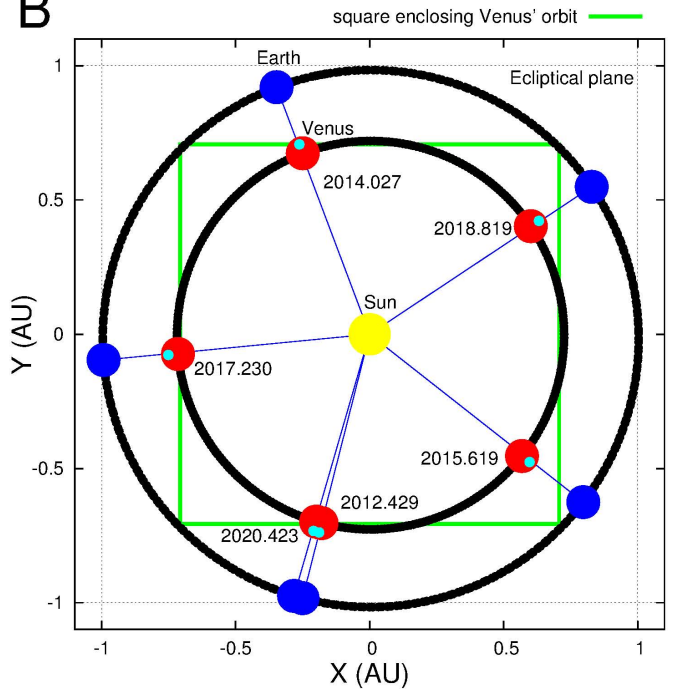

Figure 1. (A) Earth and Venus' orbits and their positions on 1 January for the years 2012 to 2020 in Copernicus' heliocentric system. The figure shows that every $8 \mathrm{yr}$ the Venus-Earth configuration approximately repeats forming eight-point star pattern. (B) Earth-Venus inferior conjunctions from 2012 to 2020. The figure shows a five-point star pattern. Note that at every conjunction, the same side of Venus (represented by a small cyan circle) faces Earth. The orbits and the coordinates (in astronomical units) of the planets were determined using the JPL's HORIZONS Ephemeris system (http://ssd.jpl.nasa.gov/horizons.cgi).

easy to calculate that at every inferior conjunction (that is, every time the Sun, Venus and Earth line up), the same side of Venus faces Earth (Goldreich and Peale, 1966a; Jelbring, 2013); the Venus-Earth synodic period is 583.924 days and there are five inferior conjunctions in $8 \mathrm{yr}$. In fact, as Fig. 1b shows, in one synodic period Earth revolves 1.59867 times around the Sun, while Venus rotates on its own axis 2.40277 times in the opposite direction. The sum of the fractional part of the two numbers is almost exactly $1(\sim 1.00144)$. Thus, not only is Earth almost synchronized with Venus in a $8: 13$ orbital resonance and in a $8: 5$ synodic resonance but, despite the large distance separating the two planets, it seems to have also synchronized Venus' rotation. It is unlikely that this phenomenon is just a coincidence.

Earth always sees the same face of the Moon. The lunar rotation has been synchronized with Earth by tidal torque. At least 34 moons of the solar system (e.g., the Galilean moons of Jupiter) are rotationally synchronized with their planet (http://en.wikipedia.org/wiki/Synchronous_rotation). Charon and Pluto are also gravitationally locked and keep the same face toward each other. Mercury's rotation period (58.646 days) is exactly 2/3 of its orbital period (87.969 days) (Goldreich and Peale, 1966b; Jelbring, 2013). The synchronization of Mercury's rotation with its orbital period may be due to the combined effect of the strong tidal torque by the Sun and to Mercury's eccentricity $(\sim 0.2)$, which implies that at perihelion Mercury is about $2 / 3$ of its aphelion distance from the Sun: 0.307 AU versus 0.467 AU. It is also well known that the three inner moons of Jupiter - Ganymede, Europa and Io - participate in a $1: 2: 4$ orbital resonance.
However, the synchronous rotation of Venus with the Earth's orbit is surprising, given the large distance between the two planets. In fact, the theoretical tidal elongation caused by the Earth's gravity on Venus is just a fraction of millimeter. At the inferior conjunction the tidal elongation caused by Earth on Venus is maximum and is about $3 m_{\mathrm{Ea}} R_{\mathrm{Ve}}^{4} / 2 m_{\mathrm{Ve}} d_{\mathrm{VE}}^{3}=$ $0.035 \mathrm{~mm}$, where $m_{\mathrm{Ea}}=1$ and $m_{\mathrm{Ve}}=0.815$ are the masses of Earth and Venus in Earth's mass unit, $R_{\mathrm{Ve}}=6051.8 \mathrm{~km}$ is the radius of Venus and $d_{\mathrm{VE}}=41.4 \times 10^{6} \mathrm{~km}$ is the average distance between Earth and Venus at the inferior conjunction.

Numerous other examples of strong commensurabilities among the planets of the solar system have been found, and some of them will be discussed in this paper (cf. Jelbring, 2013; Tattersall, 2013). Furthermore, the 27.3 days sidereal orbital period of the Moon around Earth appears well synchronized with the 27.3 days period of the Carrington rotation of the Sun, as seen from the Earth, which determines a main electromagnetic oscillation of the heliospheric current sheet in a Parker spiral. The collective synchronization among all celestial bodies in our solar system indicates that they interact energetically with each other and have reached a quasi-synchronized dynamical state.

Indeed, the bodies of the solar system interact with each other gravitationally and electromagnetically, and their orbits and rotations are periodic oscillators. As discovered by Christian Huygens in the 17 th century, entrainment or synchronization between coupled oscillators requires very little energy exchange if enough time is allowed. Huygens patented the first pendulum clock and first noted that, if hung on the same wall, after a while, pendulum clocks synchronize 
to each other due to the weak physical coupling induced by small harmonic vibrations propagating in the wall (Pikovsky, 2001). Note that the solar system is about 5 billion years old, is not part of a stellar binary system, and in its history has not experienced particularly disrupting events such as collisions with other solar systems. Therefore, a certain degree of harmonic synchronization among its components should be expected.

Newtonian mechanics calculates that the theoretical tidal elongation induced by the gravity of the planets inside the Sun is just a fraction of millimeter (Scafetta, 2012c). Therefore, tidal forcing appears too small to effect the Sun. However, as discussed above, the magnitude of the tidal elongation induced by the Earth's gravity on Venus is also a fraction of millimeter. Thus, if the Earth's gravity or some other planetary mechanism has synchronized the rotation of Venus with Earth, the planets could have synchronized the internal dynamics of the Sun, and therefore they could be modulating solar activity. It seems simply unlikely that in a solar system where everything appears more or less synchronized with everything else, only the Sun should not be synchronized in some complex way with planetary motion.

Thus, the Earth's climate could be modulated by a complex harmonic forcing consisting of (1) lunar tidal oscillations acting mostly in the ocean; (2) planetary-induced solar luminosity and electromagnetic oscillations modulating mostly the cloud cover, and therefore the Earth's albedo; and (3) a gravitational synchronization with the Moon and other planets of the solar system modulating, for example, the Earth's orbital trajectory and its length of day (cf. Mörner, 2013).

From Kepler's basic concepts forward through time, this paper briefly summarizes some of the results that have further suggested the existence of a complex synchronization structure permeating the entire solar system whose physical origin is still not fully understood. A number of empirical studies have shown that a complex synchronized planetary harmonic order may characterize not only the solar planetary system but also the Sun's activity and the Earth's climate, fully confirming Kepler's vision about the existence of a harmony of the world. Preliminary physical mechanisms are being proposed as well.

This brief review is not fully comprehensive of all the results. It simply introduces a general reader to this fascinating issue. The next sections review general results found in the scientific literature showing and discussing (1) the ordered structure of the planetary system; (2) the likely planetary origin of the variability of the Sun's activity; and (3) the synchronization of the Earth's climate with lunar, planetary and solar harmonics.

\section{Kepler's vision of a cosmographic mystery}

About half of a century after Copernicus, Kepler corrected and extended the heliocentric model. Kepler found that (1) the orbit of every planet is an ellipse (instead of Copernicus' perfect cycles) with the Sun at one of the two foci (instead of being in the center of the cycle), (2) a line joining a planet and the Sun sweeps out equal areas during equal intervals of time, and (3) the square of the orbital period of a planet is proportional to the cube of the semi-major axis of its orbit. If the orbital period, $T$, is measured in years and the semi-major axis, $a$, is measured in astronomical units (AU, the average Sun-Earth distance), Kepler's third law takes the simple form of $T^{2}=a^{3}$. The first two laws were published in 1609 (Kepler, 1609), while the third law was published in 1619 (Kepler, 1619). Kepler's three laws of planetary motion were later formally demonstrated by Newton (1687) using calculus and his law of universal gravitation stating that a planet is attracted by the Sun with a force directly proportional to the product of the two masses and inversely proportional to the square of the Sun-planet distance.

However, Kepler did more than just proposing his three laws of planetary motion. Since the publication of the Mysterium Cosmographicum (The Cosmographic Mystery) Kepler (1596) noted the existence of a "marvelous proportion of the celestial spheres" referring to the "number, magnitude, and periodic motions of the heavens". Kepler found specific distance relationships between the six planets known at that time (Mercury, Venus, Earth, Mars, Jupiter and Saturn). These relationships could be understood in terms of the five Platonic solids enclosed within each other, with the outer solid being a sphere that represented the orbit of Saturn (see Fig. 2a and b).

Some of these geometrical relations are easy to notice. For example, the ratio between the Earth's orbital radius $(a=$ $1 \mathrm{AU})$ and Venus' orbital radius ( $a=0.72 \mathrm{AU})$ is approximately equal to the ratio between the diagonal and the side of a square ( $\sqrt{2} \approx 1.41$ ). Thus, Venus' orbit is approximately enclosed within a square enclosed within the Earth's orbit (see Fig. 1b). Analogously, the ratio between Saturn's orbital radius $(a=9.6 \mathrm{AU})$ and Jupiter's orbital radius $(a=5.2 \mathrm{AU})$ is approximately equivalent to the ratio between the diagonal and the side of a cube ( $\sqrt{3} \approx 1.73)$. Thus, Jupiter's orbit is approximately enclosed within a cube enclosed within Saturn's orbital sphere (see Fig. 2a).

Kepler also highlighted the existence of a $5: 2$ JupiterSaturn resonance, which had been, however, well known since antiquity (Ma'Sar, 9th century; Temple, 1998): every $\sim 60 \mathrm{yr}$ the Jupiter-Saturn configuration approximately repeats because Jupiter revolves $\sim 5$ times and Saturn $\sim 2$ times. Figure $2 \mathrm{c}$ shows Kepler's original diagram of the great conjunctions of Saturn and Jupiter, which occur every $\sim 20 \mathrm{yr}$, from 1583 to 1723 . Every three conjunctions (a trigon) Jupiter and Saturn meet approximately at the same location of the zodiac, which happens every $\sim 60 \mathrm{yr}$. The 


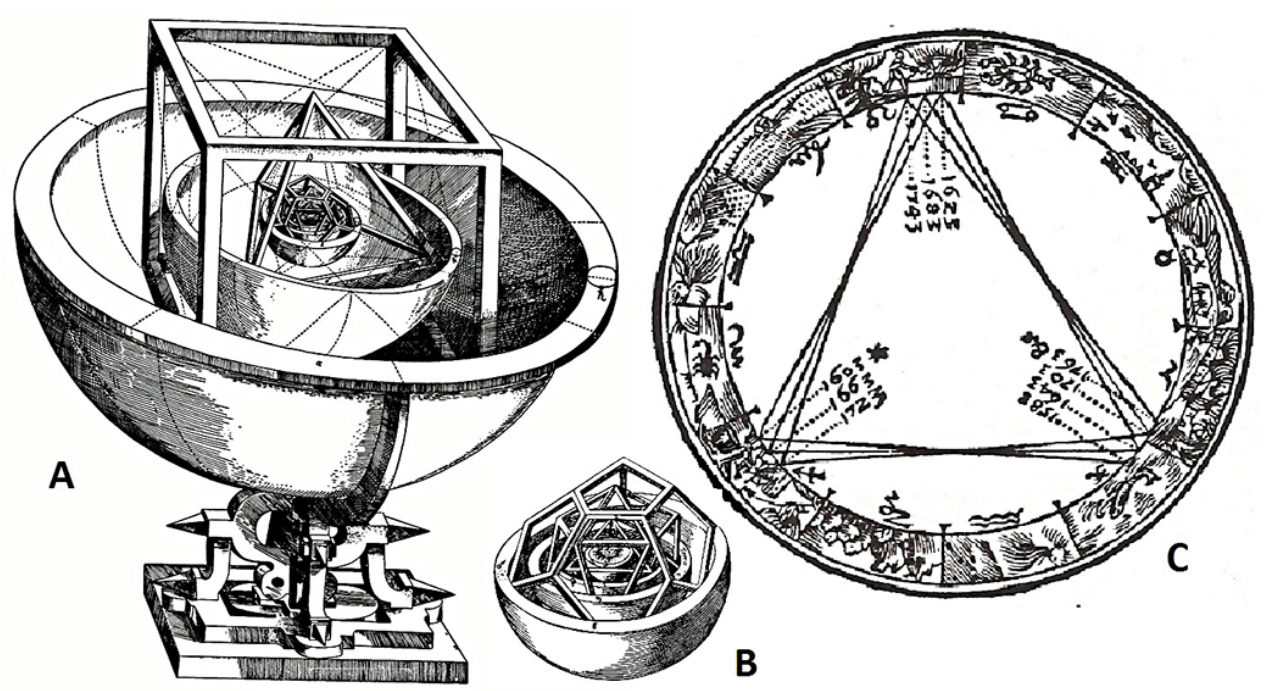

Figure 2. (A) Encapsulated Platonic solid model of the solar planetary system (Kepler, 1596). (B) Detailed view of the inner sphere. (C) A series of great conjunctions of Jupiter and Saturn from 1583 to 1723 by Kepler (1606). The figure demonstrates that every (A) 60 yr the Jupiter-Saturn configuration approximately repeats. Every $\sim 20 \mathrm{yr}$ a Jupiter-Saturn conjunction occurs. (Figures are adapted from http: //en.wikipedia.org.)

trigon slightly rotates and the configuration repeats every 800-1000 yr.

The discovery of a geometrical relationship among the semi-major axes of the planets and the relationship between the planets' orbital semi-major axis and their orbital period (the third law of planetary motion) convinced Kepler (1619) that the planetary orbits are mutually synchronized as though the solar system formed a kind of celestial choir. The great advantage of the heliocentric model was mostly to make it far easier to see this ordered structure.

Kepler also conjectured that celestial harmonics could permeate the entire solar system, including the Earth's climate (Kepler, 1601, 1606, 1619). However, modern physics would require that for the planets to modulate the Earth's climate, they first need to modulate the Sun's activity. In fact, the Sun is the most likely place where the weak planetary harmonics could be energetically amplified by a large factor. This issue will be discussed in Sects. 7 and 8 .

\section{The planetary rhythm of the Titius-Bode rule}

Titius (1766) and later Bode (1772) noted that the semi-major axes $a_{n}$ of the planets of the solar system are function of the planetary sequence number $n$. Adding 4 to the series 0,3 , $6,12,24,48,96,192$ and 384 and dividing the result by 10 gives a series that approximately reproduces the semi-major axis length of the planets in astronomical units ( 1 AU = SunEarth average distance). The Titius-Bode rule for the orbital semi-major axis length, $a_{n}$, is a power-law equation that can be written as

$a_{n}=0.4+0.3 \times 2^{n}$
Table 1. Predictions of the Titius-Bode rule against the observations. The semi-major axes $a$ are measured in astronomical units. The observed semi-major axes are from http://nssdc.gsfc.nasa.gov/ planetary/factsheet/.

\begin{tabular}{lcrrr}
\hline Planet & $n$ & $\begin{array}{r}\text { Titius-Bode rule } \\
a_{n}(\mathrm{AU})\end{array}$ & $\begin{array}{r}\text { Observations } \\
a(\mathrm{AU})\end{array}$ & Percent error \\
\hline Mercury & $-\infty$ & 0.40 & 0.387 & $(3.3 \%)$ \\
Venus & 0 & 0.70 & 0.723 & $(3.18 \%)$ \\
Earth & 1 & 1.00 & 1.00 & $(0 \%)$ \\
Mars & 2 & 1.60 & 1.524 & $(5.0 \%)$ \\
Ceres & 3 & 2.80 & 2.77 & $(1.1 \%)$ \\
Jupiter & 4 & 5.20 & 5.204 & $(0.1 \%)$ \\
Saturn & 5 & 10.00 & 9.582 & $(4.4 \%)$ \\
Uranus & 6 & 19.60 & 19.201 & $(2.1 \%)$ \\
Neptune & $?$ & $?$ & 30.047 & $?$ \\
Pluto & 7 & 38.80 & 39.482 & $(1.7 \%)$ \\
\hline
\end{tabular}

with $n=-\infty, 0,1,2,3,4,5,6,7$, where $n=-\infty$ refers to Mercury, $n=0$ to Venus, $n=1$ to Earth, etc. As Table 1 shows, the Titius-Bode empirical rule successfully predicts the orbital semi-major axis length for all the planets and dwarf planets except for Neptune.

When the Titius-Bode rule was proposed (1766-1772) the dwarf planet Ceres (in the asteroid belt) and the Jovian planet Uranus were unknown. Indeed, the idea that undiscovered planets could exist between the orbits of Mars and Jupiter and beyond Saturn was strongly suggested by Bode in 1772 . The curious gap separating Mars and Jupiter had, however, already been noted by Kepler.

The astronomers looked for new planets taking into account the predictions of the Titius-Bode rule. In 1781 
Herschel (Dreyer, 1912) discovered Uranus, and in $1801 \mathrm{Pi}-$ azzi (1801) discovered the dwarf planet Ceres. Both Ceres and Uranus fit the predictions of the Titius-Bode rule relatively well.

In the early 19th century, following Herschel and Piazzi's discoveries, the Titius-Bode rule became widely accepted as a "law" of nature. However, the discovery of Neptune in 1846 created a severe problem because its semi-major axis length $a_{\mathrm{Ne}}=30.047$ AU does not satisfy the Titius-Bode prediction for $n=7, a_{7}=38.80 \mathrm{AU}$. The discovery of Pluto in 1930 confounded the issue still further. In fact, Pluto's semi-major axis length, $a_{\mathrm{pl}}=39.482 \mathrm{AU}$, would be inconsistent with the Titius-Bode rule unless Pluto is given the position $n=7$ that the rule had predicted for Neptune (see Table 1).

The Titius-Bode rule is clearly imperfect or incomplete and no rigorous theoretical explanation of it still exists. However, it is unlikely that the relationship among the planets of the solar system that it approximately models is purely coincidental. Very likely any stable planetary system may satisfy a Titius-Bode-type relationship due to a combination of orbital resonance and shortage of degrees of freedom. Dubrulle and Graner (1994a, b) have shown that Titius-Bode-type rules could be a consequence of collapsing-cloud models of planetary systems possessing two symmetries: rotational invariance and scale invariance.

\section{The asteroid belt "mirror" symmetry rule}

Following the discovery of Ceres in 1801, numerous asteroids were discovered at approximately the same orbital distance. The region in which these asteroids were found lies between Mars and Jupiter and it is known as the asteroid belt. No planet could form in this region because of the gravitational perturbations of Jupiter that has prevented the accretion of the asteroids into a small planet. Ceres, with its spherical shape of $\sim 500 \mathrm{~km}$ radius, is the largest asteroid and the only dwarf planet in the inner solar system.

A curious mathematical relationship linking the four terrestrial inner planets (Mercury, Venus, Earth and Mars) and the four giant gaseous outer planets (Jupiter, Saturn, Uranus and Neptune) exists (Geddes and King-Hele, 1983). The semi-major axes of these eight planets appear to reflect about the asteroid belt. This mirror symmetry associates Mercury with Neptune, Venus with Uranus, Earth with Saturn and Mars with Jupiter. Geddes and King-Hele (1983) found that the mutual relations among the planets could all be approximately given as relations between the mean frequency notes in an octave: $b=2 \exp (1 / 8)$.

For example, using the semi-major axis lengths reported in Table 1 for the eight planets and labeling these distances with the first two letters of the planet's name, it is easy to obtain

$$
\begin{aligned}
\mathrm{Me} \times \mathrm{Ne} & =1.214 \cdot \mathrm{Ea} \times \mathrm{Sa} \\
\mathrm{Ve} \times \mathrm{Ur} & =1.194 \cdot \mathrm{Me} \times \mathrm{Ne} \\
\mathrm{Ea} \times \mathrm{Sa} & =1.208 \cdot \mathrm{Ma} \times \mathrm{Ju}
\end{aligned}
$$

where we have $b^{2} \approx 1.19$, and

$$
\begin{aligned}
\mathrm{Ve} \times \mathrm{Ma} & =2.847 \cdot \mathrm{Me} \times \mathrm{Ea} \\
\mathrm{Sa} \times \mathrm{Ne} & =2.881 \cdot \mathrm{Ju} \times \mathrm{Ur},
\end{aligned}
$$

where we have $b^{12} \approx 2.83$. Combining the equations yields

$$
\frac{\mathrm{Me} \times \mathrm{Ne}}{\mathrm{Ea} \times \mathrm{Sa}} \approx \frac{\mathrm{Ve} \times \mathrm{Ur}}{\mathrm{Me} \times \mathrm{Ne}} \approx \frac{\mathrm{Ea} \times \mathrm{Sa}}{\mathrm{Ma} \times \mathrm{Ju}}
$$

and

$\frac{\mathrm{Me} \times \mathrm{Ea}}{\mathrm{Ve} \times \mathrm{Ma}} \approx \frac{\mathrm{Ju} \times \mathrm{Ur}}{\mathrm{Sa} \times \mathrm{Ne}}$.

These relations relate the four inner and the four outer planets of the solar system. Even if the Geddes and King-Hele rule is not perfect, it does suggest the existence of a specific ordered structure in the planetary system where the asteroid belt region acts as a kind of mirroring boundary condition between the inner and outer regions of the solar system.

Geddes and King-Hele (1983) concluded that "the significance of the many near-equalities is very difficult to assess. The hard-boiled may dismiss them as mere playing with numbers; but those with eyes to see and ears to hear may find traces of something far more deeply interfused in the fact that the average interval between the musical notes emerges as the only numerical constant required - a result that would surely have pleased Kepler."

\section{The matrix of planetary resonances}

Molchanov (1968, 1969a) showed that the periods of the planets could be approximately predicted with a set of simple linear equations based on integer coefficients describing the mutual planetary resonances. Molchanov's system is reported below:

$$
\left(\begin{array}{ccccccccc}
1 & -1 & -2 & -1 & 0 & 0 & 0 & 0 & 0 \\
0 & 1 & 0 & -3 & 0 & -1 & 0 & 0 & 0 \\
0 & 0 & 1 & -2 & 1 & -1 & 1 & 0 & 0 \\
0 & 0 & 0 & 1 & -6 & 0 & -2 & 0 & 0 \\
0 & 0 & 0 & 0 & 2 & -5 & 0 & 0 & 0 \\
0 & 0 & 0 & 0 & 1 & 0 & -7 & 0 & 0 \\
0 & 0 & 0 & 0 & 0 & 0 & 1 & -2 & 0 \\
0 & 0 & 0 & 0 & 0 & 0 & 1 & 0 & -3
\end{array}\right)\left(\begin{array}{c}
\omega_{\mathrm{Me}} \\
\omega_{\mathrm{Ve}} \\
\omega_{\mathrm{Ea}} \\
\omega_{\mathrm{Ma}} \\
\omega_{\mathrm{Ju}} \\
\omega_{\mathrm{Sa}} \\
\omega_{\mathrm{Ur}} \\
\omega_{\mathrm{Ne}} \\
\omega_{\mathrm{Pl}}
\end{array}\right)=\left(\begin{array}{l}
0 \\
0 \\
0 \\
0 \\
0 \\
0 \\
0 \\
0
\end{array}\right),(6)
$$

where $\omega=T^{-1}$ is the orbital frequency corresponding to the planetary period $T$. By imposing $\omega_{\mathrm{Ea}}^{-1}=T_{\mathrm{Ea}}=1 \mathrm{yr}$ the system 
(Eq. 6) predicts the following orbital periods:

$\begin{array}{cllccc}\text { period } & & & \text { calculated } & \text { observed } & \text { error } \\ T_{\mathrm{Me}} & =2484 / 10332 & = & 0.240 & 0.241 & (0.4 \%) \\ T_{\mathrm{Ve}}=2484 / 4044 & = & 0.614 & 0.615 & (0.2 \%) \\ T_{\mathrm{Ea}}=1 & = & 1.000 & 1.000 & (0.0 \%) \\ T_{\mathrm{Ma}}=2484 / 1320 & = & 1.880 & 1.880 & (0.0 \%) \\ T_{\mathrm{Ju}}=2484 / 210 & = & 11.83 & 11.86 & (0.3 \%) \\ T_{\mathrm{Sa}}=2484 / 84 & = & 29.57 & 29.46 & (0.4 \%) \\ T_{\mathrm{Ur}}=2484 / 30 & = & 82.80 & 84.01 & (1.4 \%) \\ T_{\mathrm{Ne}}=2484 / 15 & = & 165.6 & 164.8 & (0.5 \%) \\ T_{\mathrm{Pl}}=2484 / 10 & = & 248.4 & 248.1 & (0.1 \%),\end{array}$

where the last column gives the observed orbital periods of the planets in years. The absolute percent divergence between the predicted and observed orbital periods is given in parentheses.

Using simple linear algebra, the system (Eq. 6) can also be used to find alternative resonance relations. For example, summing the first two rows gives the following relation between Mercury, Earth, Mars and Jupiter: $\omega_{\mathrm{Me}}-2 \omega_{\mathrm{Ea}}-4 \omega_{\mathrm{Ma}}-$ $\omega_{\mathrm{Sa}}=0$.

Molchanov (1968) showed that analogous tables of integers work also for describing planetary satellite systems such as the moon systems of Jupiter and Saturn. The provided physical explanation was that the resonant structure in a gravitationally interacting oscillating system could be inevitable under the action of dissipative perturbations of mutually comparable size. However, Molchanov (1969a) noted that alternative resonance relations yielding slightly different results could also be formulated. Nevertheless, even if it is the case that the system (Eq. 6) is neither unique nor perfectly descriptive of the orbital characteristics of the planets of the solar system, it does suggest that the planets are mutually synchronized. Molchanov (1969b) quantitatively evaluated that the probability of formation of a given resonant structure by chance is not very likely: the probability that the resonant structure of the solar system could emerge as a random chance was calculated to be less than $p=10^{-10}$.

\section{The gravitational harmonics of the solar system}

The simplest way to determine whether the solar system is characterized by a harmonic order is to study its natural frequencies and find out whether they obey some general rule. The main set of frequencies that characterize the solar planetary system can be found by studying the power spectra of physical measures that are comprehensive of the motion of all planets such as the functions describing the dynamics of the Sun relative to the center of mass of the solar system. In fact, the Sun is wobbling around the center of mass of the solar system following a very complex trajectory due to the gravitational attraction of all planets. Figure 3 shows the wobbling of the Sun during specific periods.

Several functions of the coordinates of the Sun relative to the center of mass of the solar system can be chosen such as the distance, the speed, the angular momentum, etc. (e.g.,
Jose, 1965; Bucha et al., 1985). However, simple mathematical theorems establish that generic functions of the orbits of the planets must by necessity share a common set of planetary frequencies. Only the amplitudes associated with each harmonic are expected to depend on the specific chosen observable. Thus, unless one is interested in a given observable for a specific purpose, any general function of the orbits of the planets should suffice to determine the main harmonic set describing the planetary motion of the solar system as a whole.

Herein I extend the frequency analysis of the Sun's motion made in Bucha et al. (1985) and Scafetta (2010). The JPL's HORIZONS Ephemeris system is used to calculate the speed of the Sun relative to the center of mass of the solar system from 12 December 8002 BC to 24 April 9001 AD (100-day steps). Power spectra are evaluated using the periodogram and the maximum entropy method (Press et al., 1997).

Figure $4 \mathrm{a}$ depicts the result and highlights the main planetary frequencies of the solar system. Slightly different values may be found using different observables and subintervals of the analyzed period because of statistical variability and because of the relative amplitude of the frequencies' change with the specific function of the planets' orbits that are chosen for the analysis. An estimate of the statistical theoretical error associated with each measured frequency could be obtained using the Nyquist theorem of the Fourier analysis and it is given by $\nabla f= \pm 1 / 2 L$, where $L=17003 \mathrm{yr}$ is the length of the analyzed time sequence. Thus, if $P_{0}$ is the central estimate of a period, its range is given by $P \approx P_{0} \pm P_{0}^{2} / 2 L$ (cf. Tan and Cheng, 2012).

Several spectral peaks can be recognized, such as the $\sim 1.092 \mathrm{yr}$ period of the Earth-Jupiter conjunctions; the $\sim 9.93$ and $\sim 19.86 \mathrm{yr}$ periods of the Jupiter-Saturn spring (half synodic) and synodic cycles, respectively; the $\sim 11.86$, $\sim 29.5, \sim 84$ and $\sim 165 \mathrm{yr}$ orbital period of Jupiter, Saturn, Uranus and Neptune, respectively; the $\sim 61 \mathrm{yr}$ cycle of the tidal beat between Jupiter and Saturn; and the periods corresponding to the synodic cycle between Jupiter and Neptune $(\sim 12.8 \mathrm{yr})$, Jupiter and Uranus $(\sim 13.8 \mathrm{yr})$, Saturn and Neptune $(\sim 35.8 \mathrm{yr})$, Saturn and Uranus $(\sim 45.3)$, and Uranus and Neptune $(\sim 171.4 \mathrm{yr})$, as well as many other cycles including the spring (half-synodic) periods. Additional spectra peaks at $\sim 200-220, \sim 571, \sim 928$ and $\sim 4200 \mathrm{yr}$ are also observed. Clustered frequencies are typically observed. For example, the ranges 42-48 yr, 54-70 yr, 82-100 yr (Gleissberg cycle) and 150-230 yr (Suess-de Vries cycle) are clearly observed in Fig. 4 and are also found among typical main solar activity and aurora cycle frequencies (Ogurtsov et al., 2002; Scafetta and Willson, 2013a). The subannual planetary harmonics together with their spectral coherence with satellite total solar irradiance records and other solar records are discussed in Scafetta and Willson (2013b, c), and are not reported here.

The curious fact is that the numerous spectral peaks observed in the solar motion do not seem to be randomly distributed. They could be approximately reproduced using a 

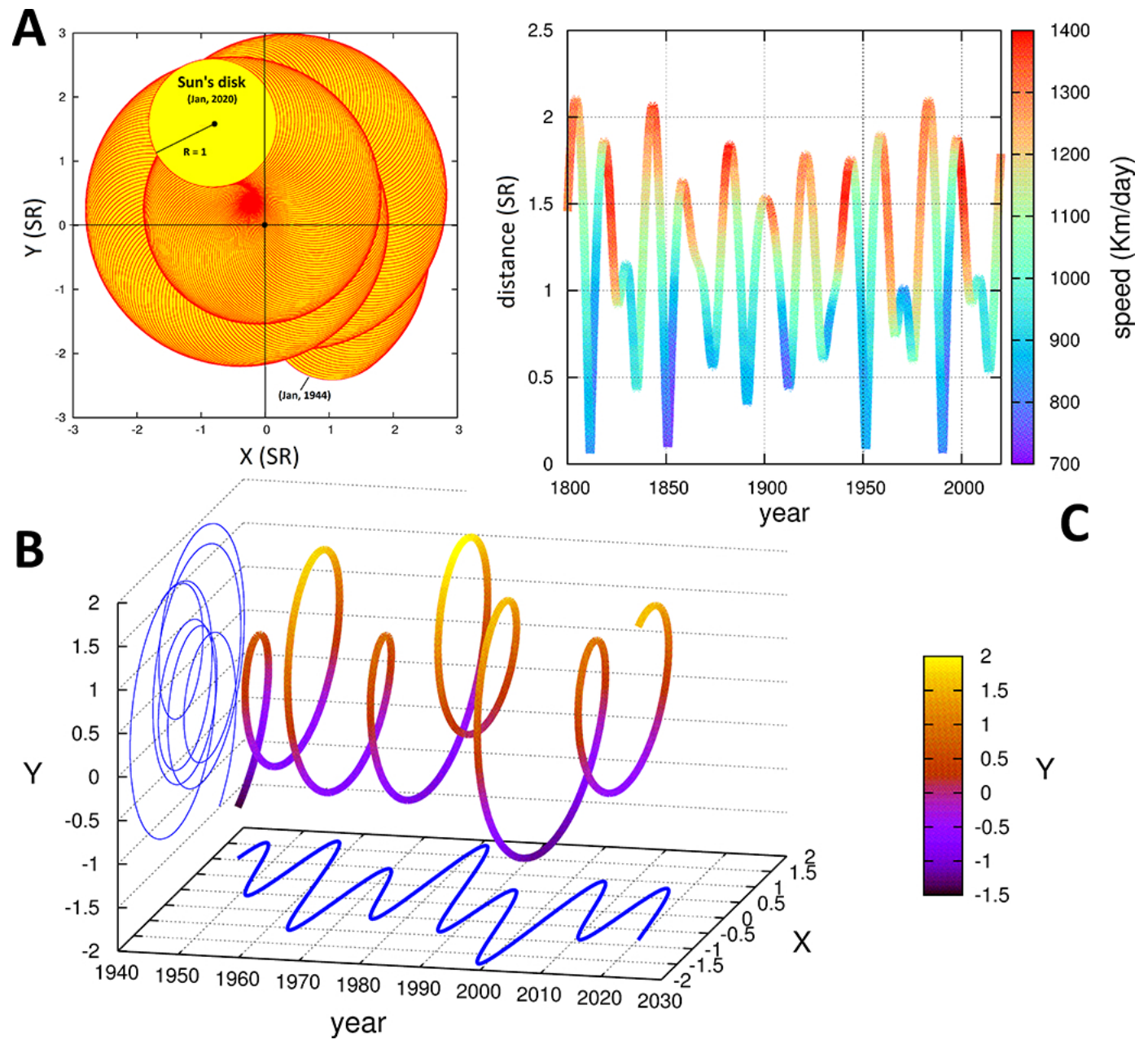

Figure 3. The wobbling of the Sun relative to the center of mass of the solar system. (A) Monthly scale movement of the Sun from 1944 to 2020 as seen from the $z$ axis perpendicular to the ecliptic. The Sun is represented by a moving yellow disk with a red circumference (cf. Ebner, 2011). (B) The trajectory of the center of the Sun from 1944 to 2020. (C) The distance and the speed of the Sun from 1800 to 2020: note the evident $\sim 20 \mathrm{yr}$ oscillation and the less evident $\sim 60$ and $\sim 170 \mathrm{yr}$ oscillation. The Sun's coordinates are estimated using the Jet Propulsion Lab's (JPL) HORIZONS Ephemeris system. The coordinates are expressed in solar radius (SR) units.

simple empirical harmonic formula of the type (Jakubcová and Pick, 1986)

$p_{i}=178.38 / i$ yr, $\quad i=1,2,3, \ldots$,

where the basic period of $\sim 178.38 \mathrm{yr}$ is approximately the period that Jose (1965) found in the Sun's motion and in the sunspot record (cf. Charvátová and Hejda, 2014). A comparison between the observed frequencies and the prediction of the resonance model, Eq. (8), is shown in Fig. 4b.

Although Eq. (8) is not perfect, and not all the modeled frequencies are clearly observed in Fig. 4a, the good agreement observed between most of the observed periods and the harmonic model predictions suggests that the solar system is characterized by a complex synchronized harmonic structure. Jakubcová and Pick (1986) also noted that several spectral peaks in the solar motion approximately correspond to the periods of various solar and terrestrial phenom- ena suggesting that the Sun itself, and the Earth's climate, could be modulated by the same planetary harmonics (see also Charvátová and Hejda, 2014). This issue is further discussed below.

\section{The planetary synchronization and modulation of the $\sim 11 \mathrm{yr}$ solar cycle}

In the 19th century, solar scientists discovered that sunspot activity is modulated by a quasi-11 yr oscillation called the Schwabe cycle. In a letter to Mr. Carrington, Wolf (1859) proposed that the observed solar oscillation could be caused by the combined influence of Venus, Earth, Jupiter and Saturn upon the Sun.

The planetary theory of solar variation is today not favored among solar scientists because, according to Newtonian 


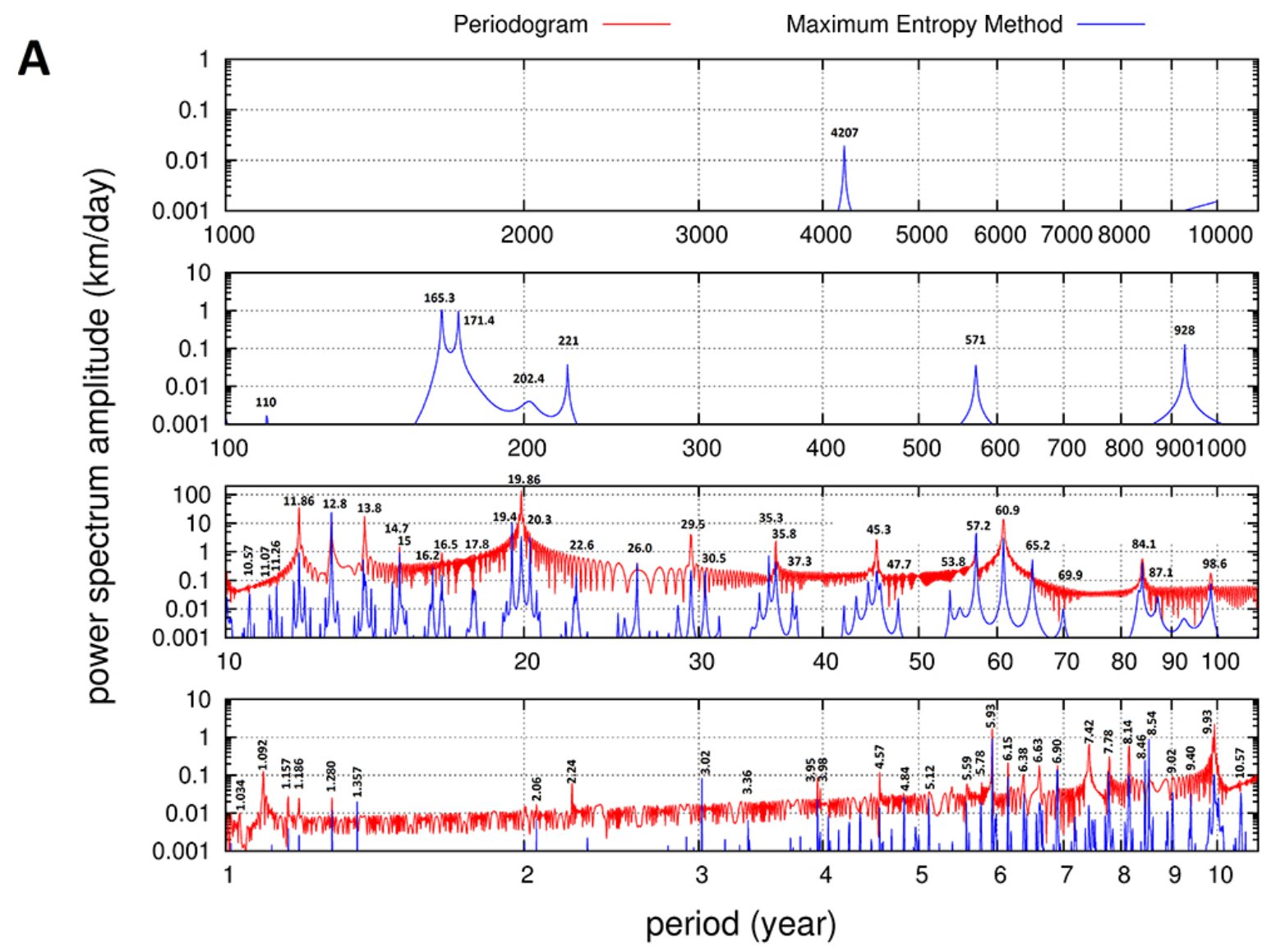

B

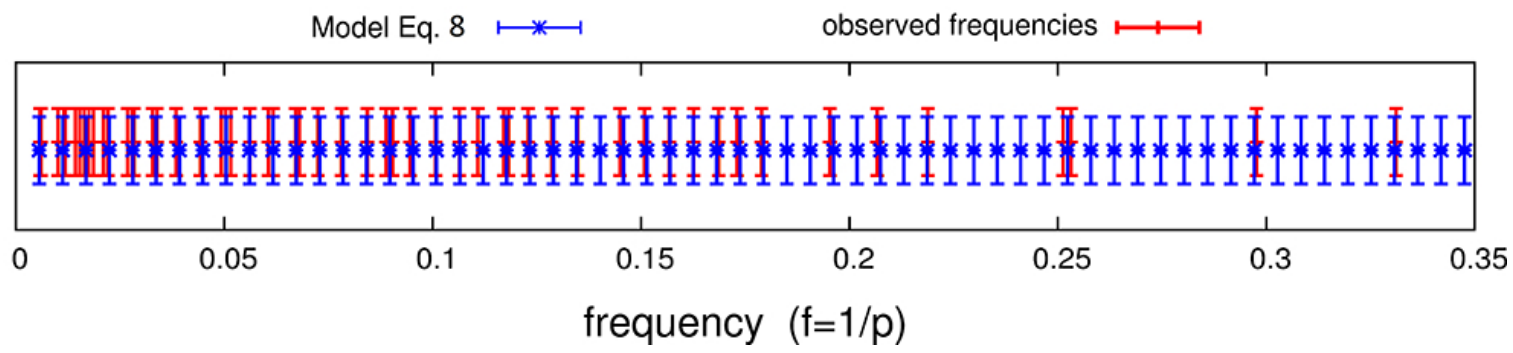

Figure 4. (A) Periodogram (red) and the maximum entropy method (blue) of the speed of the Sun relative to the center of mass of the solar system from Dec 128002 BC to 24 Apr 9001 AD. For periods larger than $200 \mathrm{yr}$ the periodogram becomes unstable and is thus not shown. (B) Comparison between the frequencies observed and listed in (A) in the range 3 to $200 \mathrm{yr}$ (red) and the frequency predictions of the resonance model Eq. (8) (blue). Note the good spectral coherence of the harmonic model with the observed frequencies.

physics, the planets appear too far from the Sun to modulate its activity, for example by gravitationally forcing the Sun's tachocline (Callebaut et al., 2012). The planets could modulate solar activity only if a mechanism exists that strongly amplifies their gravitational and/or electromagnetic influence on the Sun. Scafetta (2012c) showed that a strong amplification mechanism could be derived from the mass-luminosity relation: the gravitational energy dissipated by planetary tides on the Sun was proposed to modulate the nuclear fusion rate yielding a variable solar luminosity production. It was calculated that the proposed mechanism could yield a $4 \times 10^{6}$ energetic amplification of the tidal signal. The derived oscil- lating luminosity signal could be sufficiently strong to modulate the Sun's tachocline and convective zone (cf. Abreu et al., 2012; Mörner, 2013; Solheim, 2013a). Electromagnetic interactions between the planets and the Sun via Parker's spiral magnetic field of the heliosphere, which could be modulated by functions related to the wobbling dynamics of the Sun such as its speed, jerk, etc., could also be possible in principle. Evidence for planet-induced stellar activity has been also observed in other stars (e.g., Scharf, 2010; Shkolnik et al., 2003, 2005).

It is important to stress that the contemporary view of solar science is that solar magnetic and radiant variability is 


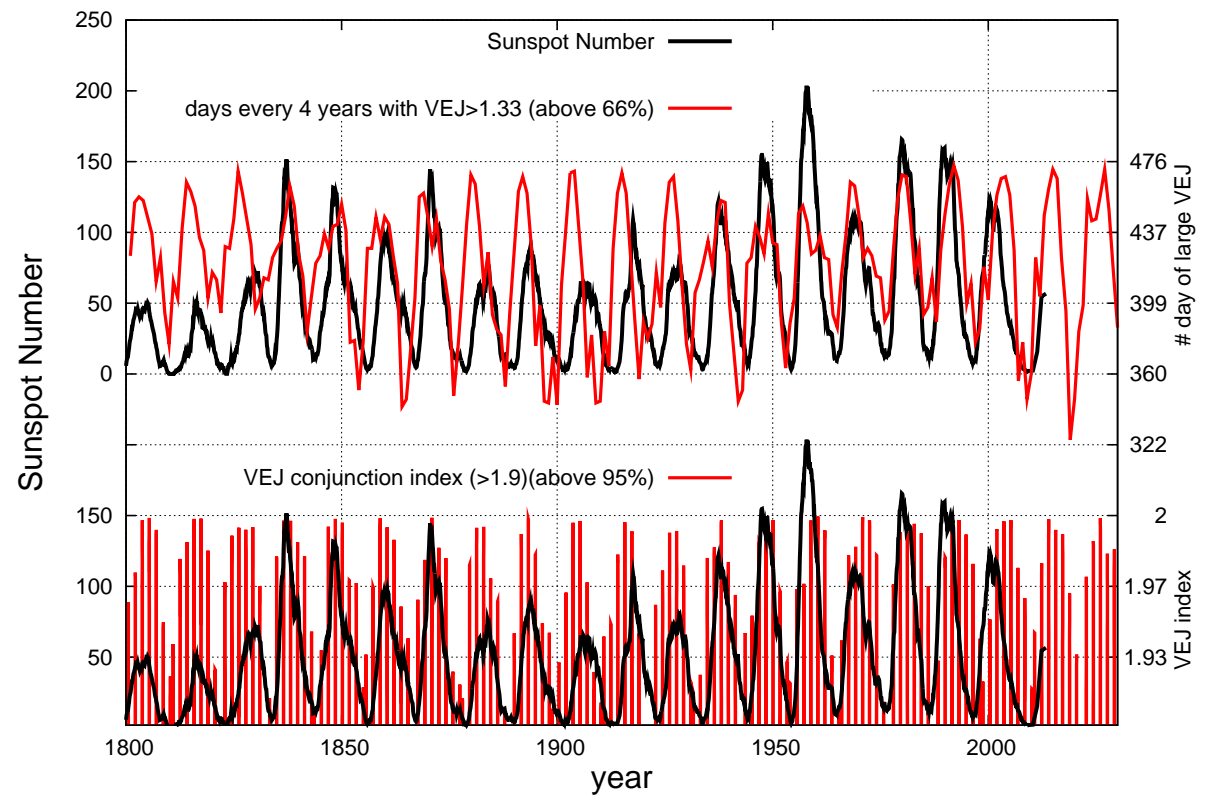

Figure 5. (Top) The sunspot number record (black) is compared against the number of days (every 4 yr) (red) when the alignment index $I_{\mathrm{VEJ}}>66 \%$. (Bottom) The sunspot number record (black) is compared against the most aligned days with $I_{\mathrm{VEJ}}>95 \%$ (red). For details see Hung (2007) and Scafetta (2012c).

intrinsically chaotic, driven by internal solar dynamics alone and characterized by hydromagnetic solar dynamo models (Tobias, 2002). However, as also admitted by solar physicists (e.g., de Jager and Versteegh, 2005; Callebaut et al., 2012), present hydromagnetic solar dynamo models, although able to generically describe the periodicities and the polarity reversal of solar activity, are not yet able to quantitatively explain the observed solar oscillations. For example, they do not explain why the Sun should present an $\sim 11$ yr sunspot cycle and $\mathrm{a} \sim 22$ yr Hale solar magnetic cycle. Solar dynamo models are able to reproduce a $\sim 11$ yr oscillation only by choosing specific values for their free parameters (Jiang et al., 2007). These dynamo models are not able to explain also the other solar oscillations observed at multiple scales such as the 50-140 yr Gleissberg cycle, the 160-260 yr Suess-de Vries cycle, the millennial solar cycles, etc. (cf. Ogurtsov et al., 2002), nor are they able to explain the phases of these cycles. Thus, the present solar dynamo theories appear to be incomplete. They cannot predict solar activity and they have not been able to explain the complex variability of the solar dynamo including the emergence of the $\sim 11 \mathrm{yr}$ oscillation. Some mechanism, which is still missed in the solar dynamo models, is needed to inform the Sun that it needs to oscillate at the observed specific frequencies and at the observed specific phases.

However, since Wolf (1859), several studies have highlighted that the complex variability of the solar dynamo appears to be approximately synchronized to planetary harmonics at multiple timescales spanning from a few days to millennia (e.g., Abreu et al., 2012; Bigg, 1967; Brown, 1900;
Charvátová, 2009; Charvátová and Hejda, 2014; Fairbridge and Shirley, 1987; Hung, 2007; Jakubcová and Pick, 1986; Jose, 1965; Scafetta, 2010, 2012a, b, c, d, 2013b; Salvador, 2013; Scafetta and Willson, 2013b, a, c; Sharp, 2013; Solheim, 2013a; Tan and Cheng, 2012; Wilson, 2013a; Wolff and Patrone, 2010; and others).

Hung (2007) also reported that 25 of the 38 largest known solar flares were observed to start when one or more tideproducing planets (Mercury, Venus, Earth, and Jupiter) were either nearly above the event positions (less than $10 \mathrm{deg}$. longitude) or at the opposing side of the Sun. Hung (2007) estimated that the probability for this to happen at random was $0.039 \%$ and concluded that "the force or momentum balance (between the solar atmospheric pressure, the gravity field, and magnetic field) on plasma in the looping magnetic field lines in solar corona could be disturbed by tides, resulting in magnetic field reconnection, solar flares, and solar storms."

As Wolf (1859) proposed, the $~ 11$ yr solar cycle could be produced by a combined influence of Venus, Earth, Jupiter and Saturn. There are two main motivations for this proposal:

1. The first model relating the $11 \mathrm{yr}$ solar cycle to the configuration of Venus, Earth and Jupiter was proposed by Bendandi (1931); later Bollinger (1952), Hung (2007) and others developed equivalent models. It was observed that Venus, Earth and Jupiter are the three major tidal planets (e.g., Scafetta, 2012c). By taking into account the combined alignment of Venus, Earth and Jupiter, it is easy to demonstrate that the gravitational configuration of the three planets repeats every 


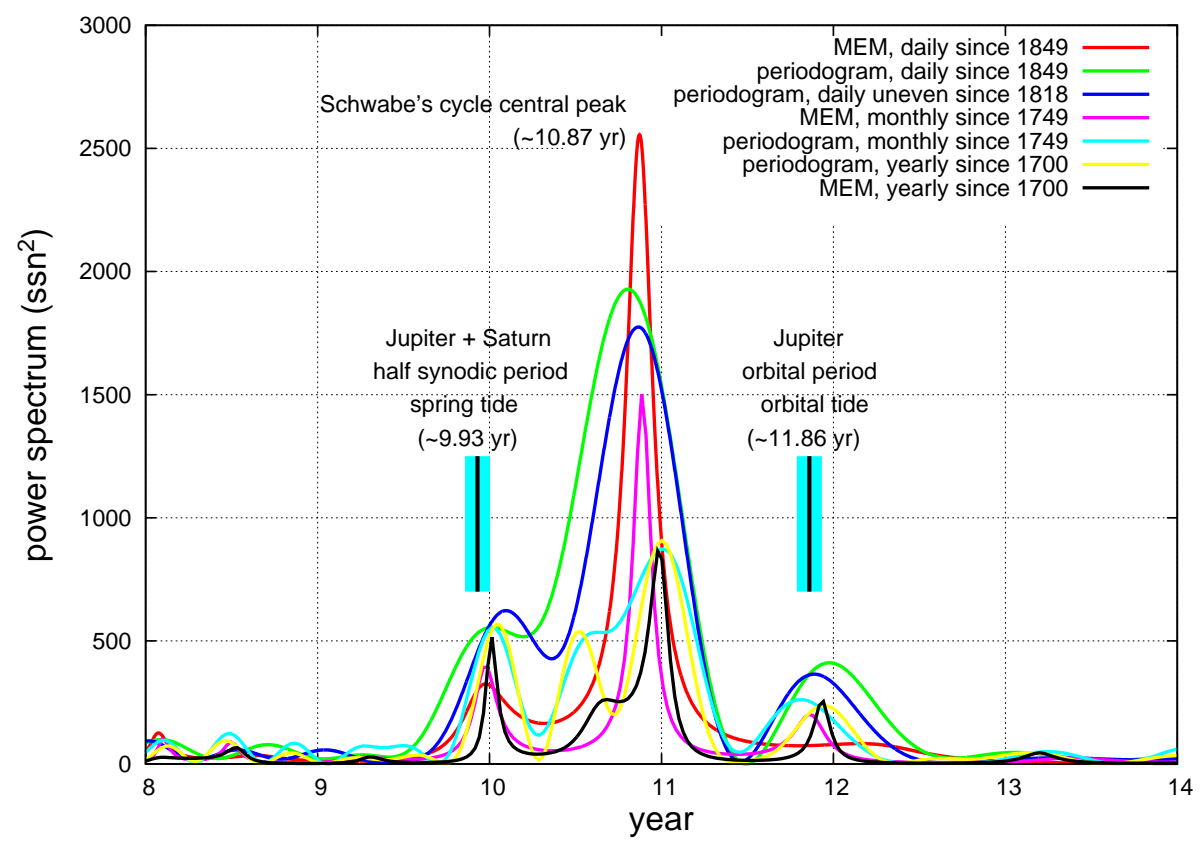

Figure 6. The three-spectral-peak structure of Schwabe's $\sim 11$ yr sunspot cycle as resolved by power spectra estimated using the maximum entropy method (MEM) and the periodogram (Press et al., 1997). The two side peaks at $\sim 9.93 \mathrm{yr}$ and $\sim 11.86 \mathrm{yr}$ correspond to the periods of Jupiter's and Saturn's spring tide and of Jupiter's orbital tide on the Sun, respectively (cf. Scafetta, 2012b, c; Solheim, 2013a). Daily, monthly and yearly resolved sunspot number records are used covering periods from 1700 to 2013: http://sidc.oma.be/sunspot-data/.

$P_{\mathrm{VEJ}}=\left(\frac{3}{P_{\mathrm{Ve}}}-\frac{5}{P_{\mathrm{Ea}}}+\frac{2}{P_{\mathrm{Ju}}}\right)^{-1}=22.14 \mathrm{yr}$

where $P_{\mathrm{Ve}}=224.701$ days, $P_{\mathrm{Ea}}=365.256$ days and $P_{\mathrm{Ju}}=4332.589$ days are the sidereal orbital periods of Venus, Earth and Jupiter, respectively (Scafetta, 2012c). The $22.14 \mathrm{yr}$ period is very close to the $\sim 22 \mathrm{yr}$ Hale solar magnetic cycle. Moreover, because the configurations $\mathrm{Ea}-\mathrm{Ve}-\mathrm{Sun}-\mathrm{Ju}$ and $\mathrm{Sun}-\mathrm{Ve}-\mathrm{Ea}-\mathrm{Ju}$ are equivalent about the tidal potential, the tidal cycle presents a recurrence of half of the above value (i.e., a period of $11.07 \mathrm{yr}$ ). This is the average solar cycle length observed since 1750 (e.g., Scafetta, 2012b). Figure 5 shows that a measure based on the most aligned days among Venus, Earth and Jupiter is well correlated, in phase and frequency, with the $\sim 11 \mathrm{yr}$ sunspot cycle: for details about the Venus-Earth-Jupiter 11.07 yr cycle see Battistini (2011, Bendandi's model), Bollinger (1952), Hung (2007), Scafetta (2012c), Salvador (2013), Wilson (2013a) and Tattersall (2013).

2. The main tides generated by Jupiter and Saturn on the Sun are characterized by two beating oscillations: the tidal oscillation associated with the orbital period of Jupiter ( $\sim 11.86 \mathrm{yr}$ period) and the spring tidal oscillation of Jupiter and Saturn $(\sim 9.93 \mathrm{yr}$ period) (Brown, 1900; Scafetta, 2012c). Scafetta (2012b, c) used detailed spectral analysis of the sunspot monthly record since 1749 and showed that the $\sim 11 \mathrm{yr}$ solar cycle is constrained by the presence of two spectral peaks close to the two theoretical tidal periods deduced from the orbits of Jupiter and Saturn (see Fig. 6). These two frequencies modulate the main central cycle at $\sim 10.87 \mathrm{yr}$ period. The beat generated by the superposition of the three harmonics is characterized by four frequencies at about $61,115,130$, and $983 \mathrm{yr}$ periods that are typically observed in solar records (e.g., Ogurtsov et al., 2002; Scafetta, 2012b). Scafetta (2012b) proposed a harmonic model for solar variability based on three frequencies at periods of $\sim 9.93, \sim 10.87$ and $\sim 11.86 \mathrm{yr}$. The phases of the three harmonics were determined from the conjunction date of Jupiter and Saturn (2000.475), the sunspot record from 1749 to 2010 (2002.364) and the perihelion date of Jupiter (1999.381), respectively. This simple three-frequency solar model not only oscillates with a $\sim 11 \mathrm{yr}$ cycle, as it should by mathematical construction, but it also manifests a complex multidecadal to millennial beat modulation that has been shown to hindcast all major patterns observed in both solar and climate records throughout the Holocene (Scafetta, 2012b). For example, the model was shown to efficiently hindcast: (1) the quasi-millennial oscillation ( $~ 983 \mathrm{yr})$ found in both climate and solar records (Bond et al., 2001); (2) the grand solar minima during the last millennium such as the Oort, Wolf, Spörer, Maunder and Dalton minima; (3) seventeen $\sim 115 \mathrm{yr}$ long oscillations found 

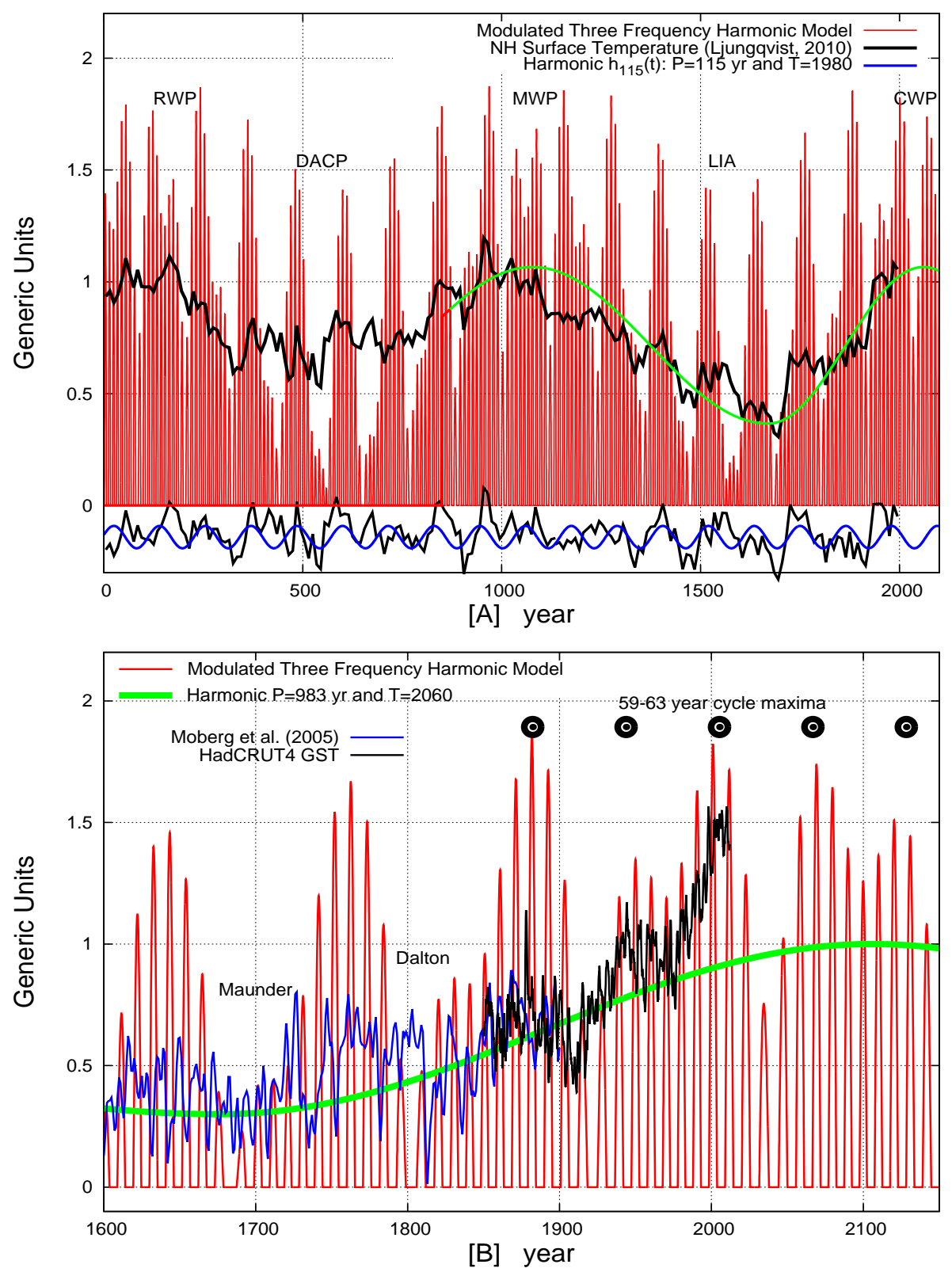

Figure 7. Scafetta (2012b) three-frequency solar model (red). (A) Against the Northern Hemisphere temperature reconstruction by Ljungqvist (2010) (black). The bottom section depicts a filtering of the temperature reconstruction (black) that highlights the $115 \mathrm{yr}$ oscillation (blue). (B) The same solar model (red) is plotted against the HadCRUT4 global surface temperature (black) merged in 1850-1900 with the proxy temperature model by Moberg et al. (2005) (blue). The green curves highlight the quasi-millennial oscillation with its skewness that approximately reproduces the millennial temperature oscillation from 1700 to 2013. Note the hindcast of the Maunder and Dalton solar minima and relative cool periods as well as the projected quasi $61 \mathrm{yr}$ oscillation from 1850 to 2150 . Adapted from Scafetta (2013a, b).

in a detailed temperature reconstruction of the Northern Hemisphere covering the last $2000 \mathrm{yr}$; and (4) the 59$63 \mathrm{yr}$ oscillation observed in the temperature record since 1850 and other features. Scafetta's (2012b) threefrequency solar model forecasts that the Sun will experience another moderate grand minimum during the following decades and will return to a grand maximum in the 2060s similar to the grand maximum experienced in the 2000s (see Fig. 7b).

Solheim (2013a) observed that if the longer sunspot yearly resolved record is used (1700-2012), then the central spectral peak observed in Fig. 6 at $\sim 10.87$ yr could be split into two peaks as $\sim 11.01 \mathrm{yr}$ and $\sim 10.66 \mathrm{yr}$ period. My own reanalysis of the periodogram of the sunspot annual record 


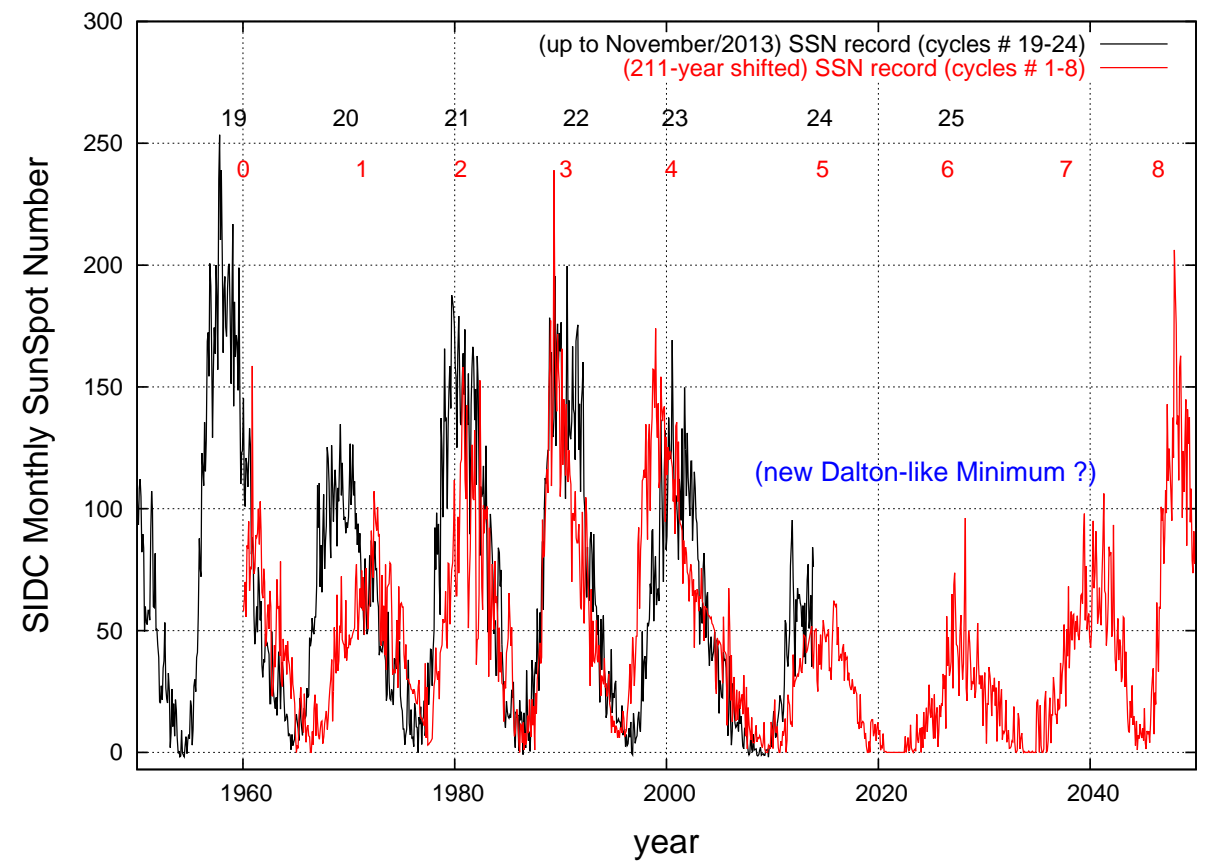

Figure 8. Comparison between latest sunspot cycles \#19-24 (black) and the sunspot cycles \#1-5 (red) immediately preceding the Dalton Minimum (1790-1830). A new Dalton-like solar minimum is likely approaching and may last until 2045 . The 211 yr temporal lag approximately corresponds to a Suess-de Vries solar cycle, which approximately corresponds to the $\sim 210$ yr beat period between the $\sim 60$ yr Jupiter-Saturn beat (Figs. 2c and 4a) and the 84 yr Uranus orbital cycle. From Scafetta (2012b).

since 1700 shows that the split produces a secondary peak at $10.52 \pm 0.2 \mathrm{yr}$ and a main peak at $11.00 \pm 0.2 \mathrm{yr}$. This result suggests that the central peak at $\sim 10.87 \mathrm{yr}$, which was interpreted in Scafetta $(2012 \mathrm{~b}, \mathrm{c})$ as being produced by an internal dynamo cycle, could indeed emerge from the Venus-EarthJupiter recurrent cycles at $\sim 11.07 \mathrm{yr}$ period plus a possible minor cycle at $\sim 10.57 \mathrm{yr}$ period. Figure 4 shows that these two spectral peaks, plus another one at $\sim 11.26 \mathrm{yr}$ period, are among the planetary harmonics. This issue needs further analysis. As for the ocean tidal system on Earth, it is possible that multiple planetary oscillations regulate the $\sim 11 \mathrm{yr}$ solar cycle.

The physical meaning of the three-frequency solar model is that solar variability at the multidecadal to millennial scales is mostly determined by the interference among the harmonic constituents that make up the main $\sim 11 \mathrm{yr}$ solar oscillation. When these harmonics interfere destructively the Sun enters into a prolonged grand minimum; when they interfere constructively the Sun experiences a grand maximum. Additional oscillations at $\sim 45, \sim 85, \sim 170$ and $\sim 210 \mathrm{yr}$ period, also driven by the other two giant planets, Uranus and Neptune (see Fig. 4), have been observed in long solar and auroral records (Ogurtsov et al., 2002; Scafetta, 2012b; Scafetta and Willson, 2013a) but not yet included to optimize the three-frequency solar model.

Note that the three-frequency solar model proposed by Scafetta (2012b) is a semi-empirical model because it is based on the two main physical tidal harmonics generated by Jupiter and Saturn plus a statistically estimated central $\sim 11 \mathrm{yr}$ solar harmonic. Therefore, this model is based on both astronomical and empirical considerations, and its hindcasting capability have been tested for both centuries and millennia. Alternative empirical models of solar variability directly based on long-range harmonics determined using power spectra and linear regressions of solar records have been also proposed (e.g., Scafetta and Willson, 2013a; Solheim, 2013a; Salvador, 2013; Steinhilber and Beer, 2013). However, models based on as many astronomical and physical considerations as possible should be preferred to purely statistical or regressive models because the former are characterized by a lower number of degrees of freedom than the latter for the same number of modeled harmonics.

The proposed semi-empirical and empirical harmonic solar models agree about the fact that the Sun is entering into a period of grand minimum. Indeed, the latest sunspot cycles \#19-24 are closely correlated to the sunspot cycles \#15 immediately preceding the Dalton Minimum (1790-1830) (see Fig. 8). Battistini (2011) noted that the $11 \mathrm{yr}$ solar cycle model proposed by Bendandi (1931) based on the VenusEarth-Jupiter configuration is slightly out of phase with both the sunspot cycles \#2-4 preceding the Dalton Minimum and with the sunspot cycles \#22-24. This result may also be further evidence suggesting that the situation preceding the 
Dalton Minimum is repeating today and could be anticipated by a planetary configuration.

\section{Astronomically based semi-empirical harmonic climate models}

As already understood since antiquity (cf. Ptolemy, 2nd century), Kepler (1601) recognized that the moon plays a crucial role in determining the ocean tidal oscillations, and in doing so, he anticipated Newton (1687) in conceiving invisible forces (gravity and electromagnetism) that could act at great distances. Kepler also argued that the climate system could be partially synchronized to multiple planetary harmonics (Kepler, 1601, 1606). The main long-scale harmonics that Kepler identified were a $\sim 20 \mathrm{yr}$ oscillation, a $\sim 60 \mathrm{yr}$ oscillation and a quasi-millennial oscillation. These oscillations were suggested by the conjunctions of Jupiter and Saturn and by historical chronological considerations (Kepler, 1606; Ma'Sar, 9th century). The quasi-millennial oscillation was associated with the slow rotation of the trigon of the conjunctions of Jupiter and Saturn, and Kepler (1606) claimed that this cycle was $\sim 800 \mathrm{yr}$ long (see Fig. 2c). Kepler's calculations were based on the tropical orbital periods of Jupiter and Saturn, which is how the orbits of Jupiter and Saturn are seen from the Earth. However, using the sidereal orbital periods this oscillation should be $850-1000 \mathrm{yr}$ long (Scafetta, 2012a), as suggested in the power spectrum analysis shown in Fig. 4. Since antiquity equivalent climatic oscillations have been noted (Iyengar, 2009; Ma'Sar, 9th century; Temple, 1998) and inserted in traditional calendars. For example, the Indian and Chinese traditional calendars are based on a $60 \mathrm{yr}$ cycle known in the Indian tradition as the Brihaspati (which means Jupiter) cycle.

The existence of climatic oscillations at about 10, 20, 60 and $1000 \mathrm{yr}$ (and others) have been confirmed by numerous modern studies analyzing various instrumental and proxy climatic records such as the global surface temperature, the Atlantic Multidecadal Oscillation (AMO), the Pacific Decadal Oscillation (PDO), the North Atlantic Oscillation (NAO), ice core records, tree ring records, sea level records, fishery records, etc. (e.g., Bond et al., 2001; Chylek et al., 2011; Klyashtorin et al., 2009; Knudsen, 2011; Jevrejeva et al., 2008; Mörner, 1989; Scafetta, 2012a, 2013c; Wyatt and Curry, 2013). Indeed, numerous authors have also noted a correlation at multiple scales between climate oscillations and planetary functions - for example, those related to the dynamics of the Sun relative to the barycenter of the solar system (e.g., Charvátová, 1997; Charvátová and Hejda, 2014; Fairbridge and Shirley, 1987; Jakubcová and Pick, 1986; Landscheidt, 1989; Scafetta, 2010, 2012b; Solheim, 2013a).

In particular, global surface temperature records, which are available from 1850 , present at least four major spectral peaks at periods of about 9.1,10-11, 20 and $60 \mathrm{yr}$, plus three minor peaks at about 12,15 and $30 \mathrm{yr}$ (see Fig. 1 in Scafetta, 2013b, which is partially reproduced in Solheim, 2013b). Subdecadal astronomical oscillations are also observed in climatic records (Scafetta, 2010). In addition, multisecular and millennial oscillations (e.g., there are major $\sim 115$ and $\sim 983$ yr oscillations and others) can be deduced from paleoclimatic proxy temperature models. As also shown in Fig. 4, these oscillations can be associated with planetary harmonics (Scafetta, 2010, 2012b). Astronomically based semi-empirical harmonic models to reconstruct and forecast climatic changes are being proposed by several authors (e.g., Abdusamatov, 2013; Akasofu, 2013; Lüdecke et al., 2013; Salvador, 2013; Scafetta, 2010, 2012a, b, d, 2013a; Solheim, 2013a).

For example, Scafetta (2013b) proposed a semi-empirical harmonic climate model based on astronomical oscillations plus an anthropogenic and volcano contribution. In its latest form this model is made of the following six astronomically deduced harmonics with periods of 9.1, 10.4, 20, 60, 115, 983 yr:

$$
\begin{aligned}
& h_{9.1}(t)=0.044 \cos (2 \pi(t-1997.82) / 9.1) \\
& h_{10.4}(t)=0.030 \cos (2 \pi(t-2002.93) / 10.4) \\
& h_{20}(t)=0.043 \cos (2 \pi(t-2001.43) / 20) \\
& h_{60}(t)=0.111 \cos (2 \pi(t-2001.29) / 60) \\
& h_{115}(t)=0.050 \cos (2 \pi(t-1980) / 115) \\
& h_{983}(t)=0.350 \cos (2 \pi(t-2060) / 760) .
\end{aligned}
$$

In the last equation a $760 \mathrm{yr}$ period from 1680 to 2060 is used instead of a $983 \mathrm{yr}$ period because the millennial temperature oscillation is skewed. While its maximum is predicted to occur in 2060, the minimum occurs around 1680 during the Maunder Minimum (1645-1715) (see Fig. 7a above and Fig. 8 in Humlum et al., 2011).

The $9.1 \mathrm{yr}$ cycle was associated with a soli-lunar tidal oscillation (e.g., Scafetta, 2010, 2012d). The rationale was that the lunar nodes complete a revolution in $18.6 \mathrm{yr}$ and the Saros soli-lunar eclipse cycle completes a revolution in $18 \mathrm{yr}$ and 11 days. These two cycles induce $9.3 \mathrm{yr}$ and $9.015 \mathrm{yr}$ tidal oscillations corresponding respectively to the Sun-EarthMoon and Sun-Moon-Earth symmetric tidal configurations. Moreover, the lunar apsidal precession completes one rotation in $8.85 \mathrm{yr}$, causing a corresponding lunar tidal cycle. The three cycles cluster between 8.85 and $9.3 \mathrm{yr}$ periods producing an average period around $9.06 \mathrm{yr}$. This soli-lunar tidal cycle peaked in 1997-1998, when the solar and lunar eclipses occurred close to the equinoxes and the tidal torque was stronger because centred on the Equator. Indeed, the $\sim 9.1 \mathrm{yr}$ temperature cycle was found to peak in 1997.82, as expected from the soli-lunar cycle model (Scafetta, 2012d).

The other five oscillations of Eq. (10) were deduced from solar and planetary oscillations. The $10.4 \mathrm{yr}$ cycle appears to be a combination of the $\sim 10 \mathrm{yr}$ Jupiter-Saturn spring cycle and the $\sim 11 \mathrm{yr}$ solar cycle and peaks in 2002.93 (i.e., $\sim 1 \mathrm{yr}$ after the maximum of solar cycle 23 ) that occurred in $\sim 2002$. The $\sim 20$ and $\sim 60 \mathrm{yr}$ temperature cycles are synchronized 


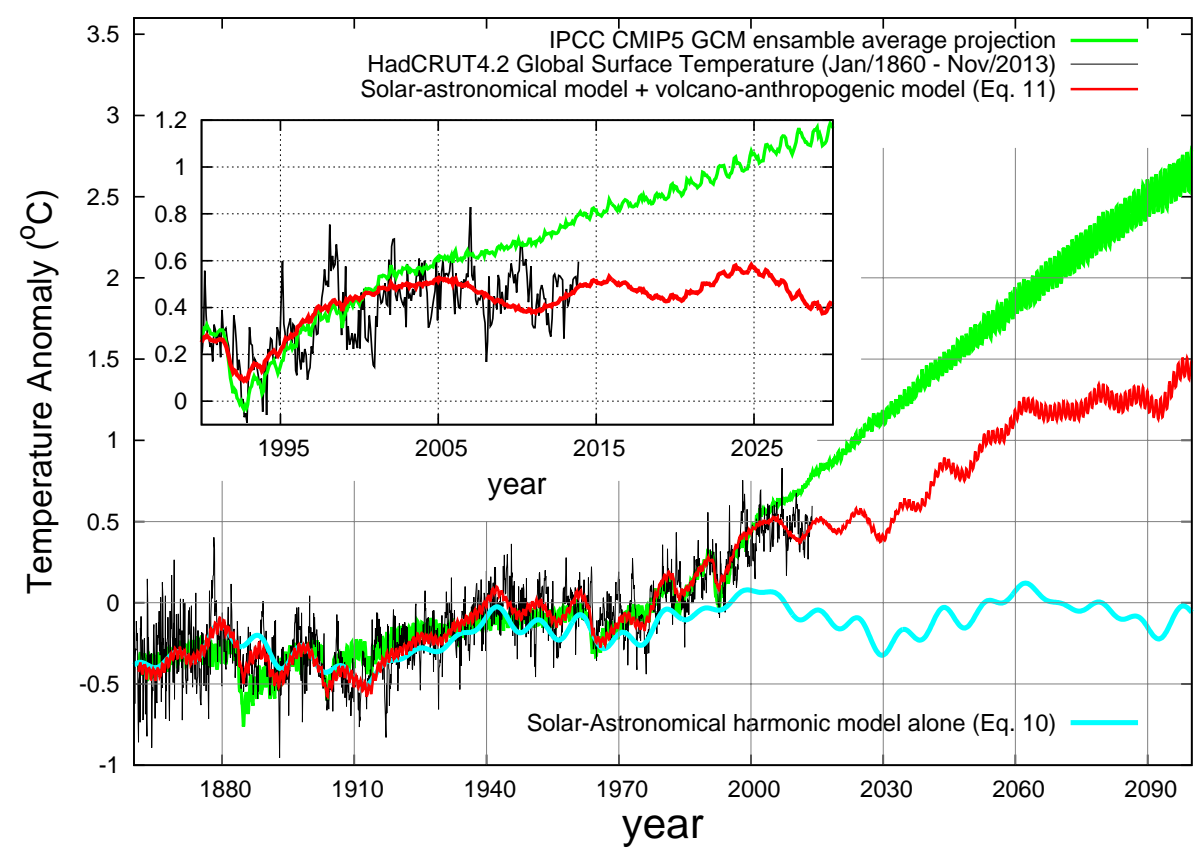

Figure 9. The semi-empirical model (Eq. 11) using $\beta=0.5$ (red) attenuation of the CMIP5 GCM ensemble mean simulation vs. HadCRUT4 GST record from Jan 1860 to Nov 2013 (black). The cyan curve represents the natural harmonic component alone (Eq. 10). The green curve represents the CMIP5 GCM average simulation used by the IPCC in 2013. The model reconstructs the 20th century warming and all decadal and multidecadal temperature patterns observed since 1860 significantly better than the GCM simulations such as the standstill since $\sim 1997$, which is highlighted in the insert (cf. Scafetta, 2010, 2012d, 2013b).

with the $\sim 20$ and $\sim 60$ yr oscillations of the speed of the Sun relative to the center of mass of the solar system (Scafetta, 2010) and the $\sim 61 \mathrm{yr}$ beat cycle of the Jupiter-Saturn tidal function, which peaked around the 1880s, 1940s and 2000s (Scafetta, 2012b, c) (see also Fig. 7b). I note, however, that Wilson (2013b) proposed a complementary explanation of the $\sim 60$ yr climatic oscillation, which would be caused by planetary induced solar activity oscillations resonating with tidal oscillations associated to specific lunar orbital variations synchronized with the motion of the Jovian planets.

The $\sim 115$ and $\sim 983$ yr oscillations are synchronized with both the secular and millennial oscillations found in climatic and solar proxy records during the Holocene (Scafetta, $2012 b)$. The amplitude of the millennial cycle is determined using modern paleoclimatic temperature reconstructions (Ljungqvist, 2010; Moberg et al., 2005). The six oscillations of Eq. (10) are quite synchronous to the correspondent astronomical oscillations (see Fig. 7 and Scafetta, 2010, $2013 b$ ). Only the amplitudes of the oscillations are fully free parameters that are determined by regression against the temperature record. See Scafetta (2010, 2012b, 2013b) for details.

To complete the semi-empirical model, a contribution from anthropogenic and volcano forcings was added. It could be estimated using the outputs of typical general circulation models (GCMs) of the Coupled Model Intercomparison Project 5 (CMIP5) simulations, $m(t)$, attenuated by half, $\beta=$
0.5 (Scafetta, 2013b). The attenuation was required to compensate for the fact that the CMIP3 and CMIP5 GCMs do not reproduce the observed natural climatic oscillations (e.g., Scafetta, 2010, 2012d, 2013b). This operation was also justified on the ground that the CMIP5 GCMs predict an almost negligible solar effect on climate change and their simulations essentially model anthropogenic plus volcano radiative effects alone. Finally, the adoption of $\beta=0.5$ was also justified by the fact that numerous recent studies (e.g., Chylek et al., 2011; Chylek and Lohmann, 2008; Lewis, 2013; Lindzen and Choi, 2011; Ring et al., 2012; Scafetta, 2013b; Singer, 2011; Spencer and Braswel, 2011; Zhou and Tung, 2012) have suggested that the true climate sensitivity to radiative forcing could be about half $\left(\sim 0.7-2.3^{\circ} \mathrm{C}\right.$ for $\mathrm{CO}_{2}$ doubling) of the current GCM estimated range $\left(\sim 1.5\right.$ to $4.5^{\circ} \mathrm{C}$; IPCC, 2013).

Scafetta's (2013b) semi-empirical climate model was calculated using the following formula:

$$
\begin{aligned}
H(t) & =h_{9.1}(t)+h_{10.4}(t)+h_{20}(t)+h_{60}(t)+h_{115}(t)+h_{983}(t) \\
& +\beta \times m(t)+\text { const. }
\end{aligned}
$$

Figure 9 shows that the model (Eq. 11, red curve) successfully reproduces all of the decadal and multidecadal oscillating patterns observed in the temperature record since 1850 , including the upward trend and the temperature standstill since 2000. However, the decadal and multidecadal temperature oscillations and the temperature standstill since $\sim 2000$ 
are macroscopically missed by the CMIP5 GCM simulations adopted by the Intergovernmental Panel on Climate Change (IPCC, 2013) (cf. Scafetta, 2013b). As Fig. 9 shows, Eq. (11) projects a significantly lower warming during the 21 st century than the CMIP5 average projection.

Alternative empirical models for the global surface temperature have been proposed by Scafetta (2010, 2012a, d, 2013a), Solheim (2013b), Akasofu (2013), Abdusamatov (2013), Lüdecke et al. (2013), Vahrenholt and Lüning (2013) and others. These models are based on the common assumption that the climate is characterized by specific quasiharmonic oscillations linked to astronomical-solar cycles. However, they differ from each other in important mathematical details and physical assumptions. These differences yield different performances and projections for the 21st century. For example, Scafetta's (2010, 2012a, d, 2013a, b) models predict a temperature standstill until the 2030s and a moderate anthropogenic warming from 2000 to 2100 modulated by natural oscillations such as the $\sim 60 \mathrm{yr}$ cycle (see the red curve in Fig. 9). Scafetta's model takes into account that the natural climatic variability, driven by a forecasted solar minimum similar to a moderate Dalton solar minimum or to the solar minimum observed during $\sim 1910$ (see Figs. 7b and 8) would yield a global cooling of $\sim 0.4{ }^{\circ} \mathrm{C}$ from $\sim 2000$ to 2030 (see cyan curve in Fig. 9), but this natural cooling would be mostly compensated by anthropogenic warming as projected throughout the 21 st century by Scafetta's $\beta$-attenuated model (see Eq. 11). Although with some differences, the climatic predictions of Solheim (2013b), Akasofu (2013) and Vahrenholt and Lüning (2013) look quite similar: they predict a steady to moderate global cooling from 2000 to 2030 and a moderate warming for 2100 modulated by a $\sim 60 \mathrm{yr}$ cycle. However, Abdusamatov (2013, Fig. 8 ) predicted an imminent cooling of the global temperature beginning from the year 2014 that will continue throughout the first half of the 21 st century and would yield a Little Ice Age period from $\sim 2050$ to $\sim 2110$, when the temperature would be $\sim 1.2^{\circ} \mathrm{C}$ cooler than the $2000-2010$ global temperature. Abdusamatov's predicted strong cooling would be induced by an approaching Maunder-like solar minimum period that would occur during the second half of the 21 st century. Steinhilber and Beer (2013) also predicted a grand solar minimum occurring during the second half of the 21 st century, but it would be quite moderate and more similar to the solar minimum observed during $\sim 1910$; thus, this solar minimum will not be as deep as the Maunder solar minimum of the 17th century.

An analysis and comparison of the scientific merits of each proposed harmonic constituent solar and climate model based on astronomical oscillations elude the purpose of this paper and it is left to the study of the reader. In general, harmonic models based only on statistical, Fourier and regression analysis may be misleading if the harmonics are not physically or astronomically justified. Nonetheless, harmonic constituent models can work exceptionally well in reconstructing and forecasting the natural variability of a system if the dynamics of the system are sufficiently harmonic and the constituent physical/astronomical harmonics are identified with great precision. For example, the astronomically based harmonic constituent models currently used to predict the ocean tides are the most accurate predictive geophysical models currently available (Doodson, 1921; http://en.wikipedia.org/wiki/Theory_of_tides).

Scafetta (2012b, d, 2013b) carefully tested his solar and climate models based on astronomical oscillations using several hindcasting procedures. For example, the harmonic solar model was tested in its ability to hindcast the major solar patterns during the Holocene and the harmonic climate model was calibrated during the period 1850-1950 and its performance to obtain the correct 1950-2010 patterns was properly tested, and vice versa. Future observations will help to better identify and further develop the most reliable harmonic constituent climate model based on astronomical oscillations.

\section{Conclusions}

Pythagoras of Samos (Pliny the Elder, 77 AD) proposed that the Sun, the Moon and the planets all emit their own unique hum (orbital resonance; cf. Tattersall, 2013) based on their orbital revolution, and that the quality of life on Earth reflects somehow the tenor of the celestial sounds (from http://en. wikipedia.org/wiki/Musica_universalis). This ancient philosophical concept is known as musica universalis (universal music or music of the spheres). However, it is with Copernicus' heliocentric revolution that the harmonic structure of the solar system became clearer. Kepler $(1596,1619)$ strongly advocated the harmonices mundi (the harmony of the world) concept from a scientific point of view.

Since the 17th century, scientists have tried to disclose the fundamental mathematical relationships that describe the solar system. Interesting resonances linking the planets together have been found. I have briefly discussed the TitiusBode rule and other resonant relationships that have been proposed during the last centuries. In addition, planetary harmonics have been recently found in solar and climate records, and semi-empirical models to interpret and reconstruct the climatic oscillations, which are not modeled by current GCMs, have been proposed (e.g., Scafetta, 2013b).

How planetary harmonics could modulate the Sun and the climate on the Earth is still unknown. Some papers have noted that a tidal-torquing function acting upon hypothesized distortions in the Sun's tachocline present planetary frequencies similar to those found in solar proxy and climate records (e.g., Abreu et al., 2012; Wilson, 2013a). However, whether planetary gravitational forces are energetically sufficiently strong to modulate the Sun's activity in a measurable way remains a serious physical problem and reason for skepticism. Also, basic Newtonian physics, such as simple evaluations of tidal accelerations on just the Sun's tachocline, does not 
seem to support the theory due to the fact that planetary tidal accelerations on the Sun seem are too small (just noise) compared to the strengths of the typical convective accelerations (Callebaut et al., 2012).

However, the small gravitational perturbation that the Sun is experiencing are harmonic, and the Sun is a powerful generator of energy very sensitive to gravitational and electromagnetic variations. Thus, the Sun's internal dynamics could synchronize to the frequency of the external forcings and it could work as a huge amplifier and resonator of the tenuous gravitational music generated by the periodic synchronized motion of the planets. Scafetta (2012c) proposed a physical amplification mechanism based on the mass-luminosity relation. In Scafetta's model the Sun's tachocline would be forced mostly by an oscillating luminosity signal emerging from the solar interior (cf. Wolff and Patrone, 2010). The amplitude of the luminosity anomaly signal driven by the planetary tides, generated in the Sun's core and quickly propagating as acoustic-like waves in the radiative zone into the Sun's tachocline, has to oscillate with the tidal and torquing planetary gravitational frequencies because function of the gravitational tidal potential energy dissipated in the solar interior. The energetic strength of this signal was estimated and found to be sufficiently strong to synchronize the dynamics of the Sun's tachocline and, consequently, of the Sun's convective zone. The quasi-harmonic and resonant structure observed in the solar system should further favor the emergence of collective synchronization patterns throughout the solar system and activate amplification mechanisms in the Sun and, consequently, in the Earth's climate.

Although a comprehensive physical explanation has not been fully found yet, uninterrupted aurora records, solar records and long solar proxy records appear to be characterized by astronomical harmonics from monthly to the millennial timescales, and the same harmonics are also present in climate records, as has been found by numerous authors since the 19th century (e.g., Wolf, 1859; Brown, 1900; Abreu et al., 2012; Charvátová, 2009; Charvátová and Hejda, 2014; Fairbridge and Shirley, 1987; Hung, 2007; Jakubcová and Pick, 1986; Jose, 1965; Salvador, 2013; Scafetta, 2010, 2012a, b, c, d, 2013b; Scafetta and Willson, 2013b, a, c; Sharp, 2013; Solheim, 2013a; Tan and Cheng, 2012; Wilson, 2011, 2013a; Wolff and Patrone, 2010). Thus, gravitational and electromagnetic planetary forces should modulate both solar activity and, directly or indirectly, the electromagnetic properties of the heliosphere. The climate could respond both to solar luminosity oscillations and to the electromagnetic oscillations of the heliosphere and synchronize to them. The electromagnetic oscillations of the heliosphere and the interplanetary electric field could directly influence the Earth's cloud system through a modulation of cosmic ray and solar wind, causing oscillations in the terrestrial albedo, which could be sufficiently large (about $1-3 \%$ ) to cause the observed climatic oscillations (e.g., Mörner, 2013; Scafetta, 2012a, 2013b; Svensmark, 2007; Tinsley, 2008; Voiculescu et al., 2013).

Although the proposed rules and equations are not perfect yet, the results of this paper do support the idea that the solar system is highly organized in some form of complex resonant and synchronized structure. However, this state is dynamical and is continuously perturbed by chaotic variability, as it should be physically expected. Future research should investigate planets-Sun and space-climate coupling mechanisms in order to develop more advanced and efficient analytical and semi-empirical solar and climate models. A harmonic set made of the planetary harmonics listed in Fig. 4 plus the beat harmonics generated by the solar synchronization (e.g., Scafetta, 2012b) plus the harmonics deducible from the solilunar tides (e.g., Wang et al., 2012) perhaps constitutes the harmonic constituent group that is required for developing advanced astronomically based semi-empirical harmonic climate models.

As Pythagoras, Ptolemy, Kepler and many civilizations have conjectured since antiquity, solar and climate forecasts and projections based on astronomical oscillations appear physically possible. Advancing this scientific research could greatly benefit humanity.

Acknowledgements. The author thanks R. C. Willson (ACRIM science team) for support, the referees for useful suggestions and the editors for having organized the special issue Pattern in solar variability, their planetary origin and terrestrial impacts (Pattern Recognition in Physics, 2013; Editors: N.-A. Mörner, R. Tattersall, and J.-E. Solheim), where 10 authors try to further develop the ideas about the planetary-solar-terrestrial interaction.

Edited by: N.-A. Mörner

Reviewed by: two anonymous referees

\section{References}

Abdusamatov, Kh. I.: Grand minimum of the total solar irradiance leads to the little ice age, Geology \& Geosciences, 28, 62-68, 2013.

Abreu, J. A., Beer, J., Ferriz-Mas, A., McCracken, K. G., and Steinhilber, F.: Is there a planetary influence on solar activity?, Astron. Astrophys., 548, A88, doi:10.1051/0004-6361/201219997, 2012.

Akasofu, S.-I.: On the Present Halting of Global Warming, Climate, 1, 4-11, 2013.

Battistini, A.: Il ciclo undecennale del sole secondo Bendandi (The 11-year solar cycle according to Bendandi). New Ice Age, http: //daltonsminima.altervista.org/?p=8669, 2011.

Bendandi, R.: Un principio fondamentale dell'Universo (A fundamental principle of the Universe). (Faenza, Osservatorio Bendandi), 1931.

Bigg, E. K.: Influence of the planet Mercury on sunspots, Astron. J., 72, 463-466, 1967.

Brown, E. W.: A Possible Explanation of the Sun-spot Period, Mon. Not. R. Astron. Soc., 60, 599-606, 1900. 
Bode, J. E.: Anleitung zur Kenntnis des gestirten Himmels, 2nd Edn., Hamburg, p. 462, 1772.

Bollinger, C. J.: A 44.77 year Jupiter-Earth-Venus configuration Sun-tide period in solar-climate cycles, Academy of Science for 1952 - Proceedings of the Oklahoma, 307-311, http://digital. library.okstate.edu/oas/oas_pdf/v33/v307_311.pdf, 1952.

Bond, G., Kromer, B., Beer, J., Muscheler, R., Evans, M. N., Showers, W., Hoffmann, S., Lotti-Bond, R., Hajdas, I., and Bonani, G.: Persistent solar influence on North Atlantic climate during the Holocene, Science, 294, 2130-2136, 2001.

Bucha, V., Jakubcová, I., and Pick, M.: Resonance frequencies in the Sun's motion, Stud. Geophys. Geod., 29, 107-111, 1985.

Callebaut, D. K., de Jager, C., and Duhau, S.: The influence of planetary attractions on the solar tachocline, J. Atmos. Sol.-Terr. Phy., 80, 73-78, 2012.

Charvátová, I.: Solar-terrestrial and climatic phenomena in relation to solar inertial motion, Surv. Geophys., 18, 131-146, 1997.

Charvátová, I.: Long-term predictive assessments of solar and geomagnetic activities made on the basis of the close similarity between the solar inertial motions in the intervals 1840-1905 and 1980-2045, New Astron., 14, 25-30, 2009.

Charvátová, I. and Hejda, P.: Responses of the basic cycle of 178.7 years and 2402 years in solar-terrestrial phenomena during Holocene, Pattern Recogn. Phys., in preparation, 2014.

Chylek, P. and Lohmann, U.: Aerosol radiative forcing and climate sensitivity deduced from the Last Glacial Maximum to Holocene transition, Geophys. Res. Lett., 35, L04804, doi:10.1029/2007GL032759, 2008.

Chylek, P., Folland, C. K., Dijkstra, H. A., Lesins, G., and Dubey, M. K.: Ice-core data evidence for a prominent near 20 year timescale of the Atlantic Multidecadal Oscillation, Geophys. Res. Lett., 38, L13704, doi:10.1029/2011GL047501, 2011.

Copernicus, N.: De revolutionibus orbium coelestium. (Johannes Petreius), 1543.

de Jager, C. and Versteegh, J. M.: Do planetary motions drive solar variability?, Sol. Phys., 229, 175-179, 2005.

Doodson, A. T.: The harmonic development of the tide-generating potential, Proc. R. Soc. Lon. Ser. A, 100, 305-329, 1921.

Dreyer, J. L. E.: The Scientific Papers of Sir William Herschel, edited by: Dreyer, J. L. E. (2 Vols., London), Vol. 1, p. xxviii, 1912.

Dubrulle, B. and Graner, F.: Titius-Bode laws in the solar system. Part I: Scale invariance explains everything, Astron. Astrophys., 282, 262-268, 1994a.

Dubrulle, B. and Graner, F.: Titius-Bode laws in the solar system. Part II: Build your own law from disk models, Astron. Astrophys., 282, 269-276, 1994b.

Ebner, J. E.: Gravity, Rosettes and Inertia, The General Science Journal, 16 October, 1-11, http://gsjournal.net/Science-Journals/ Essays/View/3700, 2011.

Fairbridge, R. W. and Shirley, J. H.: Prolonged minima and the 179year cycle of the solar inertial motion, Sol. Phys., 10, 191-210, 1987.

Geddes, A. B. and King-Hele, D. G.: Equations for Mirror Symmetries Among the Distances of the Planets, Q. J. Roy. Astron. Soc., 24, 10-13, 1983.

Goldreich, P. and Peale, S. J.: Resonant Rotation for Venus?, Nature, 209, 1117-1118, 1966a.
Goldreich P. and Peale, S. J.: Resonant Spin States in the Solar System, Nature, 209, 1178-1179, 1966b.

Humlum, O., Solheim, J.-E., and Stordahl, K.: Identifying natural contributions to late Holocene climate change, Global Planet. Change, 79, 145-156, 2011.

Hung, C.-C.: Apparent Relations Between Solar Activity and Solar Tides Caused by the Planets, NASA report/TM2007-214817, available at: http://ntrs.nasa.gov/search.jsp?R= 20070025111, 2007.

Intergovernmental Panel on Climate Change (IPCC) Fifth Assessment Report (AR5), Climate Change 2013: The Physical Science Basis, 2013.

Iyengar, R. N.: Monsoon rainfall cycles as depicted in ancient Sanskrit texts, Curr. Sci., 97, 444-447, 2009.

Jakubcová, I. and Pick, M.: The planetary system and solarterrestrial phenomena, Stud. Geophys. Geod., 30, 224-235, 1986.

Jelbring, H.: Celestial commensurabilities: some special cases, Pattern Recogn. Phys., 1, 143-146, doi:10.5194/prp-1-143-2013, 2013.

Jevrejeva, S., Moore, J. C., Grinsted, A., and Woodworth, P.: Recent global sea level acceleration started over 200 years ago?, Geophys. Res. Lett., 35, L08715, doi:10.1029/2008GL033611, 2008.

Jiang, J., Chatterjee, P., and Choudhuri, A. R.: Solar activity forecast with a dynamo model, Mon. Not. R. Astron. Soc., 381, 15271542, 2007.

Jose, P. D.: Sun's motion and sunspots, Astron. J., 70, 193-200, 1965.

Kepler, J.: Mysterium Cosmographicum (The Cosmographic Mystery), 1596.

Kepler, J.: On the more certain fundamentals of astrology. 1601, in: Proceedings of the American Philosophical Society, edited by: Brackenridge, J. B. and Rossi, M. A., 123, 85-116, 1979.

Kepler, J.: De Stella Nova in Pede Serpentarii (On the new star in Ophiuchus's foot), 1606.

Kepler, J.: Astronomia nova (New Astronomy), 1609. Translated by Donahue, W. H., Cambridge, Cambridge Univ. Pr., 1992.

Kepler, J.: Harmonices mundi (The Harmony of the World), 1619. Translated by Field, J., The American Philosophical Society, 1997.

Klyashtorin, L. B., Borisov, V., and Lyubushin, A.: Cyclic changes of climate and major commercial stocks of the Barents Sea, Mar. Biol. Res., 5, 4-17, 2009.

Knudsen, M. F., Seidenkrantz, M.-S., Jacobsen, B. H., and Kuijpers, A.: Tracking the Atlantic Multidecadal Oscillation through the last 8,000 years, Nat. Commun., 2, 178, doi:10.1038/ncomms1186, 2011.

Landscheidt, T.: Sun-Earth-Man, a mesh of cosmic oscillations, Urania Trust, 1989.

Lewis, N.: An objective Bayesian, improved approach for applying optimal fingerprint techniques to estimate climate sensitivity, J. Climate, doi:10.1175/JCLI-D-12-00473.1, 2013.

Lindzen, R. S. and Choi, Y.-S.: On the observational determination of climate sensitivity and its implications, Asia Pac. J. Atmos. Sci., 47, 377-390, 2011.

Ljungqvist, F. C.: A new reconstruction of temperature variability in the extratropical Northern Hemisphere during the last two millennia, Geogr. Ann. A, 92, 339-351, 2010. 
Lüdecke, H.-J., Hempelmann, A., and Weiss, C. O.: Multi-periodic climate dynamics: spectral analysis of long-term instrumental and proxy temperature records, Clim. Past, 9, 447-452, doi:10.5194/cp-9-447-2013, 2013.

Ma'Sar, A.: On Historical Astrology - The Book of Religions and Dynasties (On the Great Conjunctions), 9th century, Translated by Yamamoto, K. and Burnett, C., Brill Academic Publishers, 2000.

Moberg, A., Sonechkin, D. M., Holmgren, K., Datsenko, N. M., and Karlén, W.: Highly variable Northern Hemisphere temperatures reconstructed from low- and highresolution proxy data, Nature, 433, 613-617, 2005.

Molchanov, A. M.: The Resonant Structure of the Solar System: The Law of Planetary Distances, Icarus, 8, 203-215, 1968.

Molchanov, A. M.: The Reality of Resonances in the Solar System, Icarus, 11, 104-110, 1969a.

Molchanov, A. M.: Resonances in Complex Systems: A Reply to Critiques, Icarus, 11, 95-103, 1969 b.

Mörner, N.-A.: Changes in the Earth's rate of rotation on an El Nino to century basis, in: Geomagnetism and Paleomagnetism, edited by: Lowes, F. J., Collinson, D. W., Parry, J. H., Runcorn, S. K., Tozer, D. C., and Soward, A., Kluwer Acad. Publ., 45-53, 1989.

Mörner, N.-A.: Planetary beat and solar-terrestrial responses, Pattern Recogn. Phys., 1, 107-116, doi:10.5194/prp-1-107-2013, 2013.

Mörner, N.-A., Tattersall, R., and Solheim, J.-E.: Preface: Pattern in solar variability, their planetary origin and terrestrial impacts, Pattern Recogn. Phys., 1, 203-204, doi:10.5194/prp-1-203-2013, 2013.

Newton, I.: Philosophiæ Naturalis Principia Mathematica (Mathematical Principles of Natural Philosophy), 1687.

Ogurtsov, M. G., Nagovitsyn, Y. A., Kocharov, G. E., and Jungner, H.: Long-period cycles of the sun's activity recorded in direct solar data and proxies, Sol. Phys., 211, 371-394, 2002.

Piazzi, G.: Risultati delle Osservazioni della Nuova Stella (Palermo), 3-6, 1801.

Pikovsky, A., Rosenblum, M., and Kurths, J.: Synchronization, a universal concept in nonlinear science, Cambridge University Press, Cambridge, UK, 2001.

Pliny the Elder: Natural History, books I-II, 77AD, Translated by Rackham, H., Harvard University Press, 1938.

Press, W. H., Teukolsky, S. A., Vetterling, W. T., and Flannery, B. P.: Numerical Recipes in C, 2nd Edn., Cambridge University Press, 1997.

Ptolemy, C.: Tetrabiblos: on the influence of the stars, 2nd century, Translated by Ashmand, J. M., Astrology Classics, Bel Air, MD, 1980.

Ring, M. J., Lindner, D., Cross, E. F., and Schlesinger, M. E.: Causes of the global warming observed since the 19th century, Atmos. Clim. Sci., 2, 401-415, 2012.

Salvador, R. J.: A mathematical model of the sunspot cycle for the past $1000 \mathrm{yr}$, Pattern Recogn. Phys., 1, 117-122, doi:10.5194/prp-1-117-2013, 2013.

Scafetta, N.: Empirical evidence for a celestial origin of the climate oscillations and its implications, J. Atmos. Sol.-Terr. Phy., 72, 951-970, doi:10.1016/j.jastp.2010.04.015, 2010.

Scafetta, N.: A shared frequency set between the historical mid-latitude aurora records and the global surface temperature, J. Atmos. Sol.-Terr. Phy., 74, 145-163, doi:10.1016/j.jastp.2011.10.013, 2012a.

Scafetta, N.: Multi-scale harmonic model for solar and climate cyclical variation throughout the Holocene based on Jupiter-Saturn tidal frequencies plus the $11 \mathrm{yr}$ solar dynamo cycle, J. Atmos. Sol.-Terr. Phy., 80, 296-311, doi:10.1016/j.jastp.2012.02.016, 2012b.

Scafetta, N.: Does the Sun work as a nuclear fusion amplifier of planetary tidal forcing? A proposal for a physical mechanism based on the mass-luminosity relation, J. Atmos. Sol.-Terr. Phy., 81-82, 27-40, doi:10.1016/j.jastp.2012.04.002, 2012c.

Scafetta, N.: Testing an astronomically based decadal-scale empirical harmonic climate model versus the IPCC (2007) general circulation climate models, J. Atmos. Sol.-Terr. Phy., 80, 124-137, doi:10.1016/j.jastp.2011.12.005, 2012 d.

Scafetta, N.: Solar and planetary oscillation control on climate change: hind-cast, forecast and a comparison with the CMIP5 GCMs, Energy \& Environment, 24, 455-496, doi:10.1260/0958305X.24.3-4.455, 2013a.

Scafetta, N.: Discussion on climate oscillations: CMIP5 general circulation models versus a semi-empirical harmonic model based on astronomical cycles, Earth-Sci. Rev., 126, 321-357, doi:10.1016/j.earscirev.2013.08.008, 2013b.

Scafetta, N.: Multi-scale dynamical analysis (MSDA) of sea level records versus PDO, AMO, and NAO indexes, Clim. Dynam., doi:10.1007/s00382-013-1771-3, in press, 2013c.

Scafetta, N. and Willson, R. C.: Planetary harmonics in the historical Hungarian aurora record (1523-1960), Planet. Space Sci., 78, 38-44, doi:10.1016/j.pss.2013.01.005, 2013a.

Scafetta, N. and Willson, R. C.: Empirical evidences for a planetary modulation of total solar irradiance and the TSI signature of the 1.09 yr Earth-Jupiter conjunction cycle, Astrophys. Space Sci., 348, 25-39, doi:10.1007/s10509-013-1558-3, $2013 \mathrm{~b}$.

Scafetta, N. and Willson, R. C.: Multiscale comparative spectral analysis of satellite total solar irradiance measurements from 2003 to 2013 reveals a planetary modulation of solar activity and its nonlinear dependence on the $11 \mathrm{yr}$ solar cycle, Pattern Recogn. Phys., 1, 123-133, doi:10.5194/prp-1-123-2013, $2013 c$.

Scharf, C. A.: Possible Constraints on Exoplanet Magnetic Field Strengths from Planet-star Interaction, Astrophys. J., 722, 1547$1555,2010$.

Sharp, G. J.: Are Uranus \& Neptune Responsible for Solar Grand Minima and Solar Cycle Modulation?, Int. J. Astron. Astrophys., 3, 260-273, 2013.

Shirley, J. H., Sperber, K. R., and Fairbridge, R. W.: Sun's internal motion and luminosity, Sol. Phys., 127, 379-392, 1990.

Shkolnik, E., Walker, G. A. H., and Bohlender, D. A.: Evidence for Planet-induced Chromospheric Activity on HD 179949, ApJ, 597, 1092-1096, 2003.

Shkolnik, E., Walker, G. A. H., Bohlender, D. A., Gu, P.-G., and Kurster, M.: Hot Jupiters and Hot Spots: The Short- and LongTerm Chromospheric Activity on Stars with Giant Planets, ApJ, 622, 1075-1090, 2005.

Singer, S. F.: Lack of consistency between modeled and observed temperature trends, Energy Environ., 22, 375-406, 2011.

Solheim, J.-E.: The sunspot cycle length - modulated by planets?, Pattern Recogn. Phys., 1, 159-164, doi:10.5194/prp-1-159-2013, 2013a. 
Solheim, J.-E.: Signals from the planets, via the Sun to the Earth, Pattern Recogn. Phys., 1, 177-184, doi:10.5194/prp-1-177-2013, 2013b.

Spencer, R. W. and Braswell, W. D.: On the misdiagnosis of surface temperature feedbacks from variations in earth's radiant energy balance, Remote Sens., 3, 1603-1613, 2011.

Steinhilber, F. and Beer, J.: Prediction of solar activity for the next 500 years, J. Geophys. Res., 118, 1861-1867, 2013.

Svensmark, H.: Cosmoclimatology: a new theory emerges, Astron. Geophys., 48, 18-24, 2007.

Tan, B. and Cheng, Z.: The mid-term and long-term solar quasiperiodic cycles and the possible relationship with planetary motions, Astrophys. Space Sci., 343, 511-521, 2013.

Tattersall, R.: The Hum: log-normal distribution and planetarysolar resonance, Pattern Recogn. Phys., 1, 185-198, doi:10.5194/prp-1-185-2013, 2013.

Temple, R.: The Sirius mystery. Appendix IV "Why Sixty Years?", Destiny Books, Rochester, Vermont, http://www.bibliotecapleyades.net/universo/siriusmystery/ siriusmystery_appendix03.htm, 1998.

Tinsley, B. A.: The global atmospheric electric circuit and its effects on cloud microphysics, Rep. Prog. Phys., 71, 066801, doi:10.1088/0034-4885/71/6/066801, 2008.

Titius, J. D.: Betrachtung über die Natur, vom Herrn Karl Bonnet (Leipzig, 1766), pp. 7-8; transl. by Jaki, S., in: The early history of the Titius-Bode Law, Am. J. Phys., 40, 1014-1023, 1972.

Tobias, S. M.: The Solar Dynamo, Phil. Trans. A, 360, 2741-2756, 2002.
Vahrenholt, F. and Lüning, S.: The neglected Sun, why the Sun precludes climate catastrophe, Stacey International, London, 2013.

Voiculescu, M., Usoskin, I., and Condurache-Bota, S.: Clouds blown by the solar wind, Environ. Res. Lett., 8, 045032, doi:10.1088/1748-9326/8/4/045032, 2013.

Wang, Z., Wu, D., Song, X., Chen, X., and Nicholls, S.: Sun-moon gravitation-induced wave characteristics and climate variation, J. Geophys. Res., 117, D07102, doi:10.1029/2011JD016967, 2012.

Wilson, I. R. G.: Are Changes in the Earth's Rotation Rate Externally Driven and Do They Affect Climate? The General Science Journal, 3811, 1-31, 2011.

Wilson, I. R. G.: The Venus-Earth-Jupiter spin-orbit coupling model, Pattern Recogn. Phys., 1, 147-158, doi:10.5194/prp-1147-2013, 2013a.

Wilson, I. R. G.: Long-Term Lunar Atmospheric Tides in the Southern Hemisphere, The Open Atmospheric Science Journal, 7, 5176, 2013b.

Wolf, R.: Extract of a letter to Mr. Carrington, Mon. Not. R. Astron. Soc., 19, 85-86, 1859.

Wolff, C. L. and Patrone, P. N.: A new way that planets can affect the Sun, Sol. Phys., 266, 227-246, 2010.

Wyatt, M. G. and Curry, J. A.: Role for Eurasian Arctic shelf sea ice in a secularly varying hemispheric climate signal during the 20th century, Clim. Dynam., doi:10.1007/s00382-013-1950-2, in press, 2013.

Zhou, J. and Tung, K.-K.: Deducing multi-decadal anthropogenic global warming trends using multiple regression analysis, J. Atmos. Sci., 70, 3-8, doi:10.1175/JAS-D-12-0208.1, 2012. 